\title{
A Domain Wall Model for Hysteresis in Piezoelectric Materials
}

\author{
Ralph C. Smith \\ Center for Research in Scientific Computation \\ Department of Mathematics \\ North Carolina State University \\ Raleigh, NC 27695-8205 \\ rsmith@eos.ncsu.edu \\ Zoubeida Ounaies \\ ICASE, M/S 132C \\ NASA Langley Research Center \\ Hampton, VA 23681-2199 \\ z.ounaies@larc.nasa.gov
}

\begin{abstract}
This paper addresses the modeling of hysteresis and nonlinear constitutive relations in piezoelectric materials at moderate to high drive levels. Hysteresis and nonlinearities are due to the domain structure inherent to the materials and both aspects must be addressed to attain the full potential of the materials as sensors and actuators in high performance applications. The model employed here is based on theory developed in $[18,19]$ for hysteresis in general ferroelectric materials. This theory is based on the quantification of the reversible and irreversible motion of domain walls pinned at inclusions in the material. This yields an ODE model having five parameters. The relationship of the parameters to physical attributes of the materials is detailed and algorithms for determining estimates of the parameters using measured values of the coercive field, differential susceptibility and saturation properties of the materials are detailed. The accuracy of the model and its capability for the prediction of measured polarization at various drive levels is illustrated through a comparison with experimental data from PZT5A, PZT5H and PZT4 compounds. Finally, the ODE model formulation is amenable to inversion which facilitates the construction of an inverse compensator for linear control design.
\end{abstract}




\section{Introduction}

Piezoelectric materials provide the capability for designing actuators and sensors which are compact, lightweight, can be molded or constructed in a variety of configurations, and are relatively inexpensive. Hence the materials are being employed in an increasing number of structural and structural acoustic applications with uses which include active vibration control, the attenuation of structure-borne noise, micropositioning, and high performance structural drivers. The mechanisms which provide the materials with both their sensor and actuator capabilities are due to the noncentrosymmetric nature of the materials and, more specifically, to domain switching in response to applied fields or stresses. In the former case, the polar changes which occur when ions displace to align with an applied field produce the strains used to actuate the underlying structure. Conversely, the application of stresses produces deformations in the material which alter the polarization and subsequently generate the voltages measured when the materials are employed as sensors. These are the converse and direct piezoelectric effects.

As a result of the ferroelectric nature of the materials, they also exhibit varying degrees of hysteresis and nonlinear saturation effects at moderate to high drive levels as illustrated in Figure 1. As detailed in [19] and references therein, this form of hysteresis is generally attributed to the impediment of domain wall movement by material inclusions and stress nonhomogeneities inherent to the materials. In the absence of an applied field, domain walls form at these pinning sites to minimize the associated potential energy. Various experimental investigations have illustrated that at low input field levels, domain wall movement is reversible and can, at least conceptually, be attributed to the bending of domain walls $[3,5,14]$. For higher input fields, the local energy barriers associated with pinning sites are overcome and domain walls move for extended distances [3]. This translation of domain walls across pinning sites provides an irreversible mechanism contributing to the hysteresis observed in ferroelectric materials.

The hysteresis inherent to ferroelectric materials can be accommodated through a variety of techniques. The simplest means for minimizing hysteresis is to restrict the input fields or stresses to sufficiently low levels to maintain quasilinear behavior. However, this severely limits the capabilities of the materials and is not feasible in many high performance applications where the materials are advantageous. For certain control and damping applications, the deleterious effects of hysteresis can be minimized either indirectly through feedback mechanisms which maintain the system at low input levels or directly through techniques such as feedback linearization. While successful in certain regimes, both approaches are often significantly handicapped by the phase lags associated with the hysteresis loops [8, 17]. Furthermore, open loop applications which require a high degree of accuracy (e.g., micropositioning) are not typically amenable to these feedback techniques, and hysteresis must be accommodated through additional design criteria or models which can be used to compensate for the hysteresis and saturation nonlinearities.

In this paper, we consider a hysteresis model for piezoelectric materials which is based on the quantification of domain and domain wall mechanisms inherent to the materials. The model is based on the theory developed in $[18,19]$ for the general characterization of hysteresis in ferroelectric materials. We focus here on the modeling of hysteresis in the relation between the applied field and resulting polarization; the resulting strains can then be specified through linear constitutive relations in the manner described in $[18,19]$. In the first step of the model development, the hysteresisfree, or anhysteretic, relation between an applied field and the resulting polarization is quantified through three techniques. The first two anhysteretic models are empirical in nature and are used to provide initial estimates for parameters in the final hysteresis model. The third is based on the classical application of Boltzmann statistics and provides constitutive relations which are applicable at low drive levels. Hysteresis is then modeled through the characterization of the reversible and 
irreversible motion of domain walls pinned at inclusions in the material. The combination of the components provides an ODE model which incorporates the nonlinear constitutive relations and hysteresis observed in various PZT compounds at high drive levels.

While the model is based on the theory developed in $[18,19]$, the contributions of this paper are threefold. (i) The first is the extension of the theory to include nonsymmetric hysteresis loops. The resulting model contains the symmetric model developed in $[18,19]$ as a special case but accommodates the nonsymmetric effects due to poling in hard PZT materials. (ii) The second contribution is an extensive validation of the theory for commonly employed piezoelectric materials under a variety of drive levels. The model in $[18,19]$ was illustrated in the context of the relaxor ferroelectric PMNPT-BT employed at one drive level at sufficiently low temperatures for it to be ferroelectric. In this paper, the predictive capabilities of the model are illustrated by identifying parameters at one drive level and then using the resulting model to predict the PZT material behavior at other input levels. The model's capability for prediction is due to its basis in energy principles and provides it with an important advantage in broadband applications. Furthermore, this capability is illustrated for the commonly employed compounds PZT5A, PZT5H and PZT4. (iii) The third contribution of the paper is the development of a method for approximating the five required parameters in the model. This algorithm is analogous to that developed in [13] for magnetic materials and provides initial estimates for the parameters through a comparison of the model with physical attributes of the data including the coercive field, the differential susceptibility at various points, and the saturation characteristics of the material. These estimates can be employed in the final model, if sufficiently accurate, or used as initial values in a least squares fit to measured data. In combination, these contributions illustrate the applicability of the theory for piezoelectric materials and extend its practical feasibility for more general ferroelectric applications.

We note that the current model is quasistatic and isothermal in nature. Moreover, it is theoretically limited to materials in which crystalline anisotropies are not significant. While initial investigations indicate that its applicability extends beyond these regimes, such applications should be considered with caution until the underlying physics is incorporated in the models. The extensions of the theory to accommodate frequency and thermal effects as well as crystalline anisotropies are under investigation.

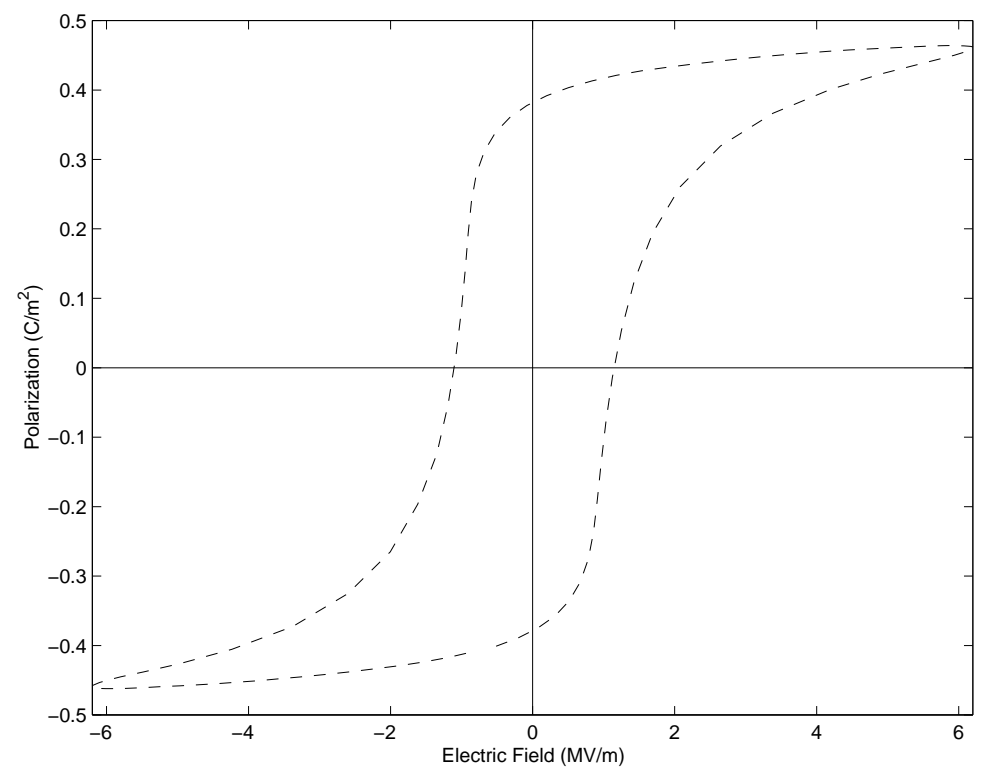

Figure 1. Hysteresis measured in a PZT5A wafer in response to a $1600 \mathrm{~V}$ input. 
A brief review of existing models for hysteresis in piezoelectric materials is summarized in the remainder of this section and the hysteresis model is then outlined in Section 2. The relationship of the model parameters to physical properties exhibited by the materials is detailed in Section 3 and an algorithm for estimating the parameters is provided. This section also includes a discussion of least squares methods which can be employed for final parameter determination. In Section 4, the model is fit to data from PZT5A, PZT5H and PZT4 wafers under a variety of drive conditions. This illustrates both the accuracy of the model and its capability for predicting the polarization due to changing drive levels.

\subsection{Existing Models}

Hysteresis models for piezoelectric materials can be roughly categorized as being microscopic, macroscopic or semi-macroscopic in nature. Microscopic theories are considered as those based on quantum principles, classical elasticity or electromagnetic relations, or thermodynamic laws applied at the lattice or grain level. While such theories are founded on the underlying physics, they often require a large number of parameters and involve states which are difficult or impossible to measure [15]. Moreover, it is difficult to incorporate attributes such as grain boundaries and intergranular stresses in the models. For this reason, microscopic models are currently limited to simple stoichiometries and are difficult to implement in control designs due to the large number of required parameters.

Macroscopic models are based on phenomenological or empirical principles and are considered advantageous when the underlying physics is poorly understood or difficult to characterize. This category includes models based on shifted anhysteretic curves which provide an envelop for the hysteresis curves (e.g., [23]) as well as various Preisach models for hysteresis in piezoelectric materials $[6,7,8]$. In addition to the generality provided by such approaches, the resulting models can for certain formulations be inverted to provide compensators for linear control design $[6,8,21]$. This has led to the fairly widespread use of Preisach techniques for modeling hysteresis in a variety of smart materials including piezoceramics. The disadvantage with this approach lies in the fact that it is difficult to employ known physics or physical measurements to directly construct the model or update the model parameters to accommodate changing operating conditions. Furthermore, to provide flexibility for a variety of drive conditions (e.g., broadband or transient conditions), it is necessary to employ a large number of nonphysical parameters which makes real-time implementation difficult.

Semi-macroscopic theories are derived using a combination of these approaches. They typically employ energy relations to characterize attributes of the polarization switching mechanism and then use macroscopic averages to obtain parameters and subsequent models for the bulk behavior of the polycrystalline material. For example, the theory of Chen and Lynch [2] employs energy relations to quantify the polarization and strain at the grain level. Macroscopic averaging over the grains is then used to characterize the aggregate behavior of the material. A model for quasistatic hysteresis was obtained by Huang and Tiersten [9] through the incorporation of internal variables in the thermodynamic state to incorporate the irreversible effects inherent to domain switching. The resulting model employs seven nonlinear material coefficients and three saturation polarization coefficients to provide a characterization of the hysteresis in the relations between the field and polarization and field and strain.

The model employed in this paper fits in the latter category. Electrostatic energy relations are employed to quantify the reversible and irreversible effects of domain wall bending and translation. Macroscopic averages then provide a model whose five parameters quantify the bulk attributes of the material. Construction in this manner provides the model with its predictive capabilities and facilitates the determination and updating of parameters from measured data. Both aspects prove 
advantageous in control applications.

This provides an overview of certain models which specifically address the modeling of hysteresis in piezoelectric materials. The reader is referred to $[18,19]$ for additional discussion of previous hysteresis models for general ferroelectric materials.

\section{Hysteresis Model}

We summarize in this section those aspects of the hysteresis theory presented in $[18,19]$ which are relevant to piezoelectric materials. In the first step of this development, various models for the anhysteretic polarization are outlined. Hysteresis is then incorporated through the quantification of the energy required to bend and translate domain walls.

\subsection{Anhysteretic Polarization}

The anhysteretic polarization curve represents the minimal energy states that would be attained by the material in the absence of inclusions or imperfections. As illustrated by the modeled curves in Figure 2, the anhysteretic has a burst region near the origin, where dipole rotation toward preferred ionic configurations produces large changes in polarization, and exhibits saturation at high field levels where charge distributions prohibit further changes. In contrast to the measured polarization curves at high drive levels, the anhysteretic polarization is single-valued and reversible.

Three techniques are outlined for modeling the anhysteretic curve. The first two are empirical and provide expressions which are used to specify parameters in the algorithms developed in Section 3. The last technique specifies the probability of dipoles occupying certain energy states through classical Boltzmann statistics. The resulting representations are used as kernels in the hysteresis model.

The first model is based on the extension of the Frölich-Kennelly anhysteretic model for magnetic materials [11, page 94] to ferroelectric materials. This yields the expression

$$
P_{a n}=\frac{\widehat{\gamma} E}{1+\gamma E}
$$

where $E$ and $P_{a n}$ respectively denote the applied electric field and anhysteretic polarization. One parameter can be eliminated by enforcing the saturation behavior $P_{a n} \rightarrow P_{s}$ as $E \rightarrow \infty$, where $P_{s}$ is the saturation polarization. This then yields the relation

$$
P_{a n}=\frac{\gamma P_{s} E}{1+\gamma E}
$$

While not employed in the final hysteresis model, the expression (1) provides a means of estimating certain parameters in the model.

A second model for the anhysteretic polarization is the empirical expression

$$
P_{a n}=\frac{\sqrt{\beta} P_{s} E}{\sqrt{1+\beta E^{2}}}
$$

developed by Piquette and Forsythe [16]. The initial behavior and slope of this curve at high fields is similar to that of (1) while the mid-range behaviors and saturation values differ. The expression (2) will also be used to specify initial parameter values in the algorithms.

The third model employs Boltzmann statistics to specify the probability of dipoles occupying certain energy states. As detailed in $[18,19]$, the balance of thermal and electrostatic energies while 
employing the assumption that the material is isotropic and the orientation of cells can be in any direction yields the Langevin equation

$$
P_{a n}=P_{s}\left[\operatorname{coth}\left(\frac{E_{e}}{a}\right)-\frac{a}{E_{e}}\right]
$$

for the anhysteretic polarization. Here

$$
E_{e}=E+\alpha P_{a n}
$$

denotes the effective field acting on dipole moments in the material while $\alpha=\widehat{E} / P_{s}$ where $\widehat{E}$ denotes a scaling electric field. The constant $a$ is specified by $a=\frac{\widehat{E} T}{3 T_{c}}$ where $T$ and $T_{c}$ are the operating and Curie temperatures for the material. Because the scaling field $\widehat{E}$ is unknown, the constants $a$ and $\alpha$ are estimated using either the algorithm derived in Section 3 or a least squares fit to data.

A second expression derived using this Boltzmann approach is the Ising spin relation

$$
P_{a n}=P_{s} \tanh \left(\frac{E_{e}}{a}\right)
$$

which results from the assumption that the dipole moments can occupy only two discrete orientations: in the direction of the applied field or opposite to it. As noted in Figure 2 where the relations (3) and (5) are compared, the restrictions on possible moment orientations causes the Ising spin relation to reach a higher saturation state than observed for the Langevin model with equivalent inputs. Further discussion concerning the merits of the two relations as well as details regarding their derivation are provided in $[18,19]$.

The relations (3) and (5), with the effective field specified by (4), yield anhysteretic curves which are rotationally symmetric about the origin as illustrated in Figure 2. For PMN-PT-BT or soft piezoceramic materials, these expressions are sufficient since the relation between the applied field and resulting polarization is also rotationally symmetric. This is not the case for hard PZT materials which have been poled, however, and the expressions must be modified to incorporate the observed asymmetries.

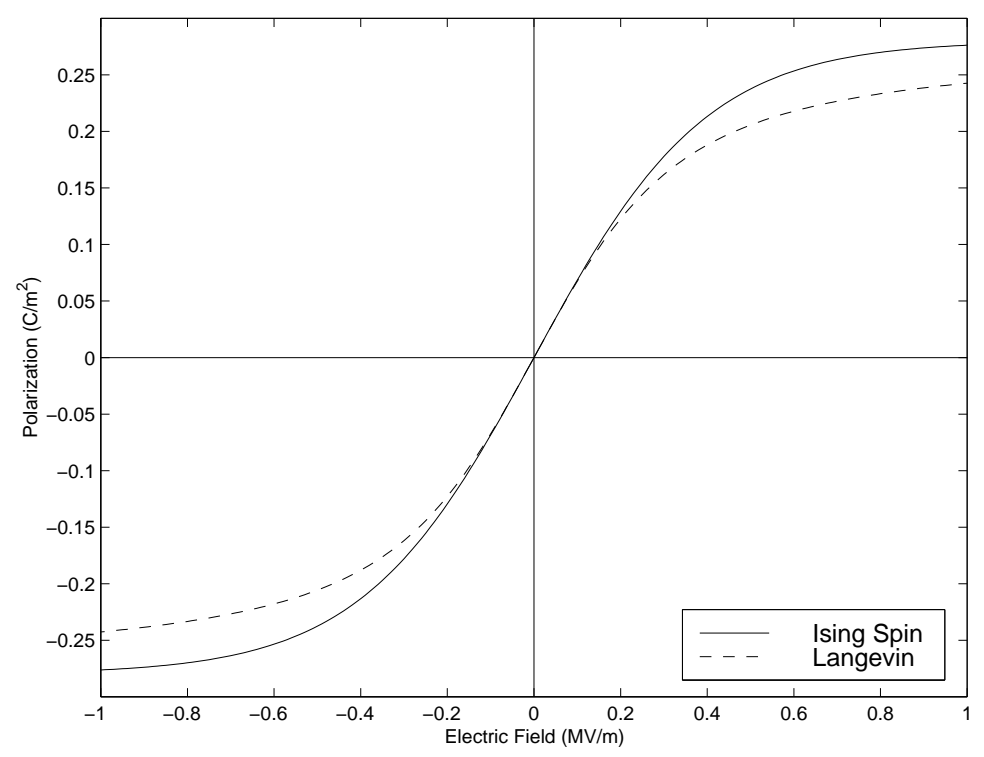

Figure 2. Ising spin and Langevin models for the anhysteretic polarization. 
To motivate modifications which incorporate the observed asymmetries, consider the data from a poled PZT4 wafer which is plotted in Figure 3. It is observed that the poling produces a bias polarization $P_{0}$ and field $E_{0}$ which causes the positive and negative remanence points and coercive fields to differ. These values bias the effective field so we consider the modified expression

$$
E_{e}=\left(E-E_{0}\right)+\alpha\left(P-P_{0}\right)
$$

In this case, the effective field reflects the bias field in the absence of an applied field $E$.

To accommodate both asymmetric major loops and biased minor loops, we also consider the modified anhysteretic expressions

$$
P_{a n}=P_{1}+P_{s}\left[\operatorname{coth}\left(\frac{E_{e}}{a}\right)-\frac{a}{E_{e}}\right]
$$

and

$$
P_{a n}=P_{1}+P_{s} \tanh \left(\frac{E_{e}}{a}\right)
$$

where $P_{1}$ is a scaling polarization. The curves produced by the Langevin model (7) with $P_{1}=0$ and $P_{1}=P_{0} \neq 0$ are illustrated in Figure 4 . In both cases, the curves are asymmetric with respect to the origin. For $P_{1}=0$, the curve is rotationally symmetric about a point $(\bar{E}, \bar{P})$ determined through the numerical solution of (7) or (8) whereas for $P_{1}=P_{0}$, the change of variables $\mathcal{P}=P-P_{0}, \mathcal{E}=E-E_{0}$ reveals that the curve is rotationally symmetric about the bias point $\left(E_{0}, P_{0}\right)$.

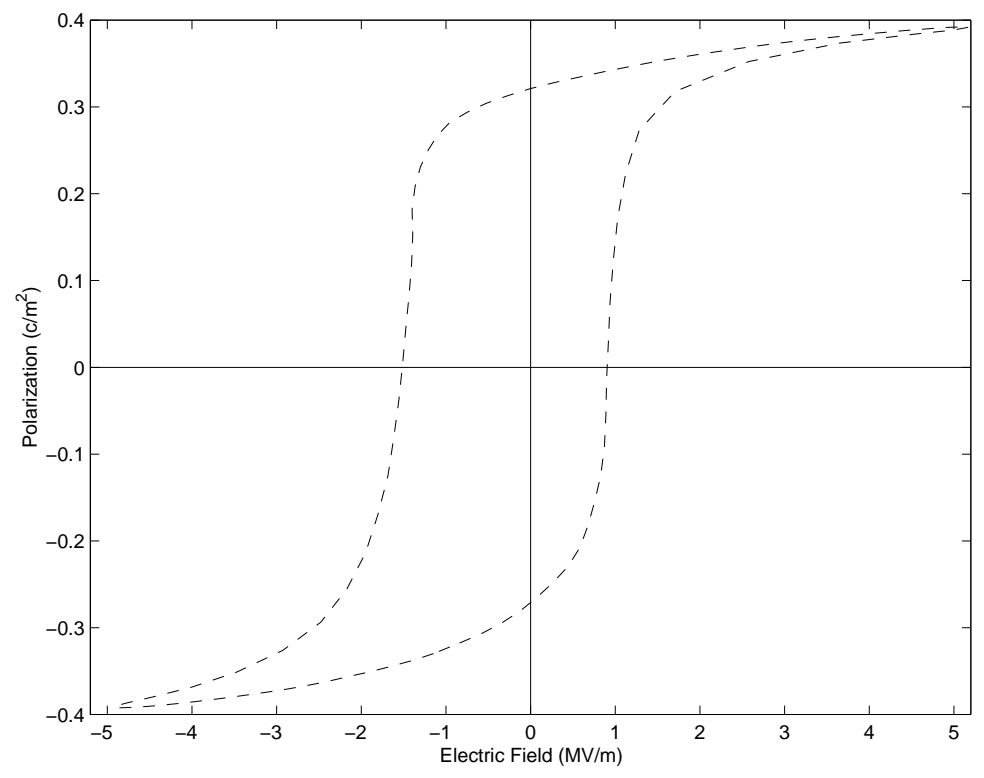

Figure 3. Data from a poled PZT4 wafer in response to a $2000 \mathrm{~V}$ input. 


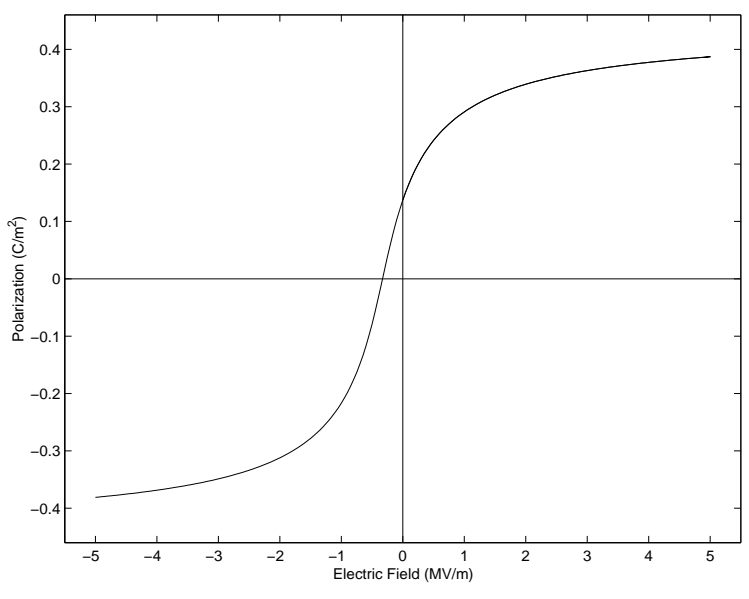

(a)

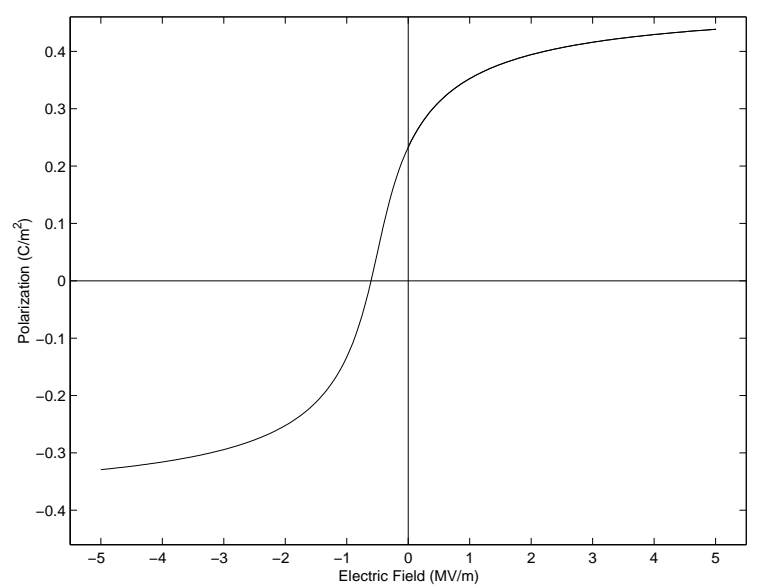

(b)

Figure 4. Anhysteretic curves produced by the Langevin model (7) with $\alpha=3.4 \times 10^{6} \mathrm{Vm} / \mathrm{C}$ and $a=8.0 \times 10^{5} \mathrm{C} / \mathrm{m}^{2}$; (a) $E_{0}=-0.5 \times 10^{6} \mathrm{~V} / \mathrm{m}, P_{0}=.05 \mathrm{C} / \mathrm{m}^{2}$ and $P_{1}=0$, (b) $E_{0}=-0.5 \times 10^{6} \mathrm{~V} / \mathrm{m}$, $P_{0}=.05 \mathrm{C} / \mathrm{m}^{2}$ and $P_{1}=P_{0}$.

\subsection{Domain Wall Model}

The relations (7) or (8) can be used to model the observed polarization at low drive levels but are inappropriate at moderate to high drive levels since they do not incorporate the hysteresis inherent to the materials. As detailed in [19] and included references, sigmoidal hysteresis of the type depicted in Figure 1 is typically attributed to the energy required to translate domain walls across pinning sites in the material. At low field levels, the walls remain close to the equilibrium position and the motion is reversible. From an energy perspective, the variations are not sufficient to cross a barrier in the potential well. The motion becomes irreversible when sufficient energy is provided to cross the potential barrier. Physically, this can occur when the domain wall intersects a remote pinning site and is the mechanism underlying domain wall translations. The resulting irreversible polarization $P_{i r r}$ and reversible polarization $P_{\text {rev }}$ are then summed to obtain the total polarization. This approach follows that employed by Jiles and Atherton in their corresponding hysteresis model for ferromagnetic materials [12].

To quantify the irreversible polarization, it is noted in $[18,19]$ that the polarization level for a given effective field can be expressed as that for the ideal case minus losses required to break pinning sites. This yields the relation

$$
P_{i r r}=P_{a n}-k \frac{d P_{i r r}}{d E_{e}}
$$

where the parameter $\delta=\operatorname{sign}(d E)$ ensures that the energy required to break pinning sites always opposes changes in polarization. The parameter $k$ is defined by $k=\frac{n\left\langle\mathcal{E}_{\pi}\right\rangle}{2 p}$ where $n$ denotes the average density of pinning sites, $\left\langle\mathcal{E}_{\pi}\right\rangle$ is the average energy for $180^{\circ}$ walls and $p$ is an average dipole moment. Because the density and energy of individual pinning sites are unknown, the parameter $k$ must be estimated for a given material.

The formulation of (9) in terms of the applied field $E$ yields the differential equation

$$
\frac{d P_{i r r}}{d E}=\frac{P_{a n}-P_{i r r}}{\delta k-\alpha\left(P_{a n}-P_{i r r}\right)}
$$


specifying the irreversible polarization. As discussed in $[18,19]$, while this expression is adequate in most regimes, it can yield nonphysical solutions when the field is reversed from saturation for materials which exhibit significant hysteresis and are driven at high levels. The enforcement of solely reversible polarization changes in this regime eliminates this discrepancy and yields the relation

$$
\frac{d P_{i r r}}{d E}=\widetilde{\delta} \frac{P_{a n}-P_{i r r}}{k \delta-\alpha\left(P_{a n}-P_{i r r}\right)}
$$

where

$$
\widetilde{\delta}=\left\{\begin{array}{l}
1,\left\{d E>0 \text { and } P<P_{a n}\right\} \text { or }\left\{d E<0 \text { and } P>P_{a n}\right\} \\
0, \text { otherwise } .
\end{array}\right.
$$

The second component of the polarization is the reversible polarization which models the effects of domain wall bending. To first approximation, this is modeled by the relation

$$
P_{\text {rev }}=c\left(P_{a n}-P_{\text {irr }}\right)
$$

where $c$ is a parameter which must be estimated for the specific application (see $[18,19]$ ).

The total polarization is then given by

$$
P=P_{\text {rev }}+P_{\text {irr }}
$$

or equivalently,

$$
P=c P_{a n}+(1-c) P_{i r r} .
$$

To implement the model, the effective field for a given field and irreversible polarization level is computed using (6). This effective field value is then employed in either (7) or (8) to compute the corresponding anhysteretic polarization. The subsequent irreversible polarization is determined by numerically integrating (10). The total polarization is then specified by (12). The determination of parameters is addressed in the next section while the prediction capabilities of the model are illustrated in Section 4.

\section{Determination of Parameters}

The implementation of the model requires the determination of the parameters $\alpha, a, k, c$ and $P_{s}$ for a given material. Recall that $\alpha$ quantifies the amount of dipole coupling in the effective field while $a$ incorporates the relative thermal effects which are balanced with the electrostatic energy to model the anhysteretic polarization. As detailed in [19], increasing $\alpha$ or decreasing $a$ leads to increased slopes in the anhysteretic curve and corresponding polarization. The parameter $k$ is a macroscopic average of the energy required to break pinning sites. Hence large $k$ values are associated with wider hysteresis loops (e.g., hard PZT materials). The parameter $c$ quantifies the average degree to which domain walls bend before translating across pinning sites; hence it too will be larger for hard materials than for soft. Finally, $P_{s}$ denotes the theoretical saturation value beyond which, polar interactions prevent further increases in polarization. This definition is in accordance with the corresponding definition for the saturation magnetization (e.g., see [11]) and should not be confused with the definition employed in several texts (e.g., [10, page 36]) for the saturation value obtained by extending the slope at tip reversal back through the vertical axis. Further details regarding the derivation and physical interpretation of these parameters are provide in [18].

In this section, we present two methods for determining the parameters based on data measurements from a given material. The first uses the measured values of the anhysteretic and initial 
susceptibilities (if available), the measured remanence polarization, the coercive field and peak tip polarization, as well as the differential susceptibilities at these points, to provide constraints which permit the determination of the parameters. This approach is analogous to that employed in [13] for magnetic materials but leads to a different algorithm for determining the parameters. This technique highlights the physical nature of the parameters and is direct to implement but can lead to model fits with limited accuracy since it employs minimal information concerning the hysteresis curve. The second approach determines the parameters through a least squares fit to the data. This provides highly accurate model fits but requires fairly good initial guesses for the parameters to reach optimal values. In practice, we employ the first technique to obtain initial parameter values. In many cases, the resulting model fit is satisfactory. If refinement is necessary, however, these parameter values can be employed as initial estimates in the least squares routine. In combination, the two approaches provide a systematic and robust means of determining the parameters for a given material.

\subsection{Direct Parameter Determination from Experimental Data}

The saturation polarization $P_{s}$ has the most direct physical connotation and can be estimated from the data at high (near saturation) drive levels. These estimates can then be refined using either of the following methods.

\subsubsection{Differential Susceptibility Relations}

To determine the constraints used to specify the remaining parameters, we consider the differential susceptibility at various points in the hysteresis cycle. From the definition (10) for the differential susceptibility of the irreversible polarization and (12) for the total polarization, we see that two cases need to be considered when computing $\frac{d P}{d E}$. When the field is first reversed from saturation, the only changes in polarization are due to the reversible effects of domain wall relaxation. This motivates the inclusion of the switch $\widetilde{\delta}$ and yields the expression

$$
\frac{d P}{d E}=c \frac{d P_{a n}}{d E}
$$

for the differential susceptibility in that region. For the remainder of the hysteresis cycle, the combination of (10) and (12) yields the expression

$$
\frac{d P}{d E}=(1-c) \frac{P_{a n}-P_{i r r}}{k \delta-\alpha\left(P_{a n}-P_{i r r}\right)}+c \frac{d P_{a n}}{d E} .
$$

A second application of the polarization relation (12) can be used to eliminate the irreversible polarization which yields

$$
\frac{d P}{d E}=(1-c) \frac{P_{a n}-P}{k \delta(1-c)-\alpha\left(P_{a n}-P\right)}+c \frac{d P_{a n}}{d E}
$$

The evaluation of (14) requires the determination of $\frac{d P_{a n}}{d E}$ which depends upon the model being employed. For the examples in Section 4, we employ the Langevin expressions (3) or (7) and we illustrate with (3) here. For general polarization values, the effective field is $E_{e}=E+\alpha P$ which yields

$$
\frac{d P_{a n}}{d E}=\frac{P_{s}}{a}\left(1+\alpha \frac{d P}{d E}\right)\left[-\operatorname{csch}^{2}\left(\frac{E+\alpha P}{a}\right)+\left(\frac{a}{E+\alpha P}\right)\right] .
$$


For the single-valued global anhysteretic curve depicted in Figure 2, the effective field is specified by (4) which yields the implicit relation

$$
\frac{d P_{a n}}{d E}=\frac{P_{s}}{a}\left(1+\alpha \frac{d P_{a n}}{d E}\right)\left[-\operatorname{csch}^{2}\left(\frac{E+\alpha P_{a n}}{a}\right)+\left(\frac{a}{E+\alpha P_{a n}}\right)\right] .
$$

\section{Initial Susceptibilities}

Taking the limits as $E \rightarrow 0, P_{a n} \rightarrow 0$, and letting $\chi_{a n}$ denote the differential susceptibility at the origin, as depicted in Figure 5, yields

$$
\chi_{a n}=\frac{P_{s}}{3 a}\left(1+\alpha \chi_{a n}\right)
$$

or

$$
\chi_{a n}=\frac{P_{s}}{3 a-\alpha P_{s}}
$$

For given $P_{s}$, this yields the expression

$$
\alpha=\frac{3 a \chi_{a n}-P_{s}}{P_{s} \chi_{a n}}
$$

relating $\alpha$ to $a$.

The second characteristic which can be employed at the origin is the initial differential susceptibility $\chi_{i n}$. Taking the limits $E \rightarrow 0, P \rightarrow 0$ in (14) yields the relation

$$
\chi_{i n}=c \chi_{a n}
$$

which can be used to specify $c$ if $\chi_{i n}$ and $\chi_{a n}$ can be measured or approximated. To evaluate the

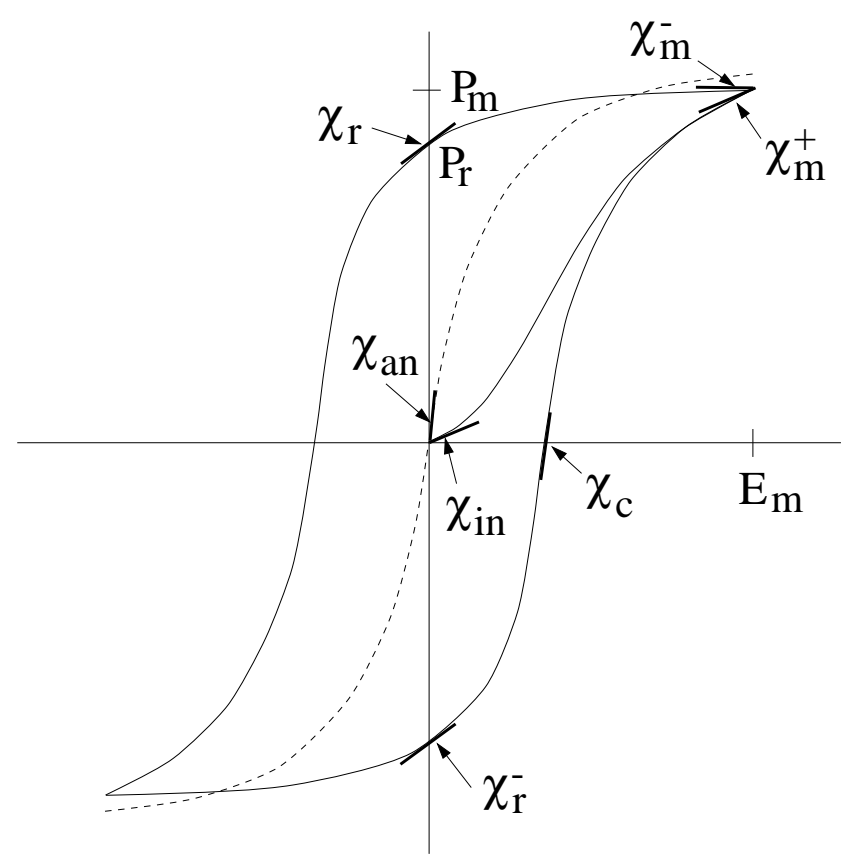

Figure 5. Hysteresis curve with differential susceptibilities employed for parameter determination. 
slope of the initial polarization curve, we take the limit $E \rightarrow 0, P \rightarrow 0$ in (14) with the anhysteretic specified by (15). This yields

$$
\chi_{i n}=\frac{P_{s} c}{3 a}\left(1+\alpha x_{i n}\right)
$$

from which it follows that

$$
\alpha=\frac{3 a \chi_{i n}-c P_{s}}{c P_{s} \chi_{i n}}
$$

Equating (17) and (19) and employing (18) to eliminate $\chi_{i n}$ then yields the expression

$$
a=\frac{P_{s}}{3 \chi_{a n}} .
$$

Once $\chi_{a n}$ has been approximated or determined from experimental data, (20) can be used to approximate the parameter $a$ and $\alpha$ can be determined from (17).

\section{Susceptibility at Field Reversal}

We consider next the behavior of the hysteresis loop near the tip value $\left(E_{m}, P_{m}\right)$. It was previously noted that directly after field reversal, the differential susceptibility is dependent only on domain wall relaxation which yields the expression (13). Furthermore, if the polarization is sufficiently close to saturation, the differential susceptibility can be approximated by

$$
\frac{d P}{d E}=\frac{d P_{a n}}{d E}
$$

in the region before field reversal (see also [13] for the magnetic case). Letting $\chi_{m}^{+}$and $\chi_{m}^{-}$respectively denote the differential susceptibilities before and after field reversal at the tip loop (see Figure 5), the combination of (13) and (21) yields the expression

$$
c=\frac{\chi_{m}^{-}}{\chi_{m}^{+}}
$$

for the reversibility coefficient. We note that the accuracy of this expression improves for measurements near saturation.

Furthermore, since $\delta=1$ before field reversal, the consideration of the approximation (21) in (14) yields

$$
\chi_{m}^{+}=\frac{P_{a n}\left(E_{m}\right)-P_{m}}{k \delta(1-c)-\alpha\left(P_{a n}\left(E_{m}\right)-P_{m}\right)}
$$

where, for the symmetric case, $P_{a n}\left(E_{m}\right)$ is specified by (3) or (5) with $E_{e}=E_{m}+\alpha P_{m}$. The expression (23) can be solved explicitly for $k$ or implicitly for $a$ or $\alpha$.

\section{Remanence Point}

Similar constraints are provided by matching the differential susceptibilities at the coercive and remanence points. At remanence, $\delta=-1, E=0$ and $P=P_{r}$ so that the relation (14) has the form

$$
\chi_{r}=(1-c) \frac{P_{a n}\left(P_{r}\right)-P_{r}}{k \delta(1-c)-\alpha\left(P_{a n}\left(P_{r}\right)-P_{r}\right)}+c \frac{d P_{a n}\left(P_{r}\right)}{d E}
$$


where, for the symmetric Langevin expression (3),

$$
\begin{aligned}
& P_{a n}\left(P_{r}\right)=P_{s}\left[\operatorname{coth}\left(\frac{\alpha P_{r}}{a}\right)-\frac{a}{\alpha P_{r}}\right] \\
& \frac{d P_{a n}}{d E}\left(P_{r}\right)=\frac{P_{s}}{a}\left(1+\alpha \chi_{r}\right)\left[-\operatorname{csch}^{2}\left(\frac{\alpha P_{r}}{a}\right)+\frac{a}{\alpha P_{r}}\right] .
\end{aligned}
$$

Because the relation (24) is implicit in the variables $a$ and $\alpha$, it is solved through either root-finding or minimization techniques.

\section{Coercive Field}

At the coercive field, $\delta=1, E=E_{c}$ and $P=0$, so the differential susceptibility is

$$
\chi_{c}=(1-c) \frac{P_{a n}\left(E_{c}\right)}{k \delta(1-c)-\alpha P_{a n}\left(E_{c}\right)}+c \frac{d P_{a n}\left(E_{c}\right)}{d E} .
$$

This can be solved for $k$ to yield

$$
k=P_{a n}\left(E_{c}\right)\left[\frac{\alpha}{1-c}+\left(\frac{1}{\chi_{c}-c \frac{d P_{a n}\left(E_{c}\right)}{d E}}\right)\right] .
$$

For specified values of $a, \alpha, c$ and $P_{s},(25)$ provides an estimate for the average energy required to break pinning sites in both hard and soft materials.

As detailed in [11, pages 170-171] for magnetic materials, this expression can be simplied significantly for soft materials when the reversible component is negligible and hence the approximation $c=0$ is valid. For materials with low coercivity, it has also been observed that the differential susceptibility at the coercive point is approximately equal to the slope of the anhysteretic curve at the origin so that

$$
\chi_{c}=\chi_{a n}
$$

(see Figure 5). Furthermore, both are approximately linear so that for small $E_{c}$, it follows from (16) that

$$
\begin{aligned}
P_{a n}\left(E_{c}\right) & =\chi_{a n} E_{c} \\
& =\left(\frac{P_{s}}{3 a-\alpha P_{s}}\right) E_{c} .
\end{aligned}
$$

We now let $c=0$ and employ the relations (26) in (25) to obtain

$$
k=\frac{E_{c}}{1-\frac{\alpha P_{s}}{3 a}} .
$$

To a first approximation, this yields the relation

$$
k \approx E_{c}
$$

As will be illustrated in the examples, the values predicted by (27) can be used as initial values for the parameter estimation routines and in many cases are quite accurate. 


\subsubsection{Approximation of $\chi_{a n}$ and $\chi_{i n}$}

The relations (16) - (25) provide constraints which will be used for constructing an algorithm for determining the parameters $a, \alpha, c$ and $k$. For cases in which the measured values of $\chi_{a n}$ and $\chi_{i n}$ are available, the constraints (16) and (18) can be applied directly. In applications in which the material starts from a poled state, however, these values may be unavailable which necessitates their approximation.

For ferromagnetic materials whose hysteresis loops are rotationally symmetric about the origin, it has been observed that for a given field value, points $M_{i n}$ on the initial magnetization curve can be approximated by the average

$$
M_{\text {in }}=\left(M_{1}+M_{2}\right) / 2
$$

where $M_{1}$ and $M_{2}$ are the corresponding magnetizations on the upper and lower hysteresis curves [1, page 511], [4]. A corresponding assumption for ferroelectric materials yields the approximation

$$
x_{i n}=\left(\chi_{r}+\chi_{r}^{-}\right) / 2
$$

where $\chi_{r}$ and $\chi_{r}^{-}$denote the differential susceptibilities at the positive and negative remanence points (see Figure 5).

The initial slope of the anhysteretic curve can be approximated using the alternative model expressions (1) and (2). To eliminate the single parameter appearing in each expression, we enforce the condition

$$
\frac{d P_{a n}}{d E}\left(P_{m}\right)=\chi_{m}
$$

where $\chi_{m}=\left(\chi_{m}^{+}+\chi_{m}^{-}\right) / 2$. In expression (1), this yields

$$
\gamma=\frac{-\left(2 E_{m} \chi_{m}-P_{s}\right)+\sqrt{P_{s}^{2}-4 P_{s} E_{m} \chi_{m}}}{2 \chi_{m} E_{m}^{2}}
$$

and the corresponding initial slope

$$
\chi_{a n_{1}}=\gamma P_{s} .
$$

The corresponding initial slope predicted by (2) is

$$
\chi_{a n_{2}}=\sqrt{\beta} P_{s}
$$

where

$$
\beta=\frac{1}{6} \frac{b^{1 / 3}}{E_{m}^{3} \chi_{m}}+\frac{2 P_{s}^{2}}{E_{m}^{3} \chi_{m} b^{1 / 3}}-\frac{1}{E_{m}^{2}}
$$

with

$$
b=-12 P_{s}^{2}\left[9 E_{m} \chi_{m}-\sqrt{3} \sqrt{-4 P_{s}^{2}+27 E_{m}^{2} \chi_{m}^{2}}\right] .
$$

The initial slope of the anhysteretic is then approximated by

$$
\chi_{a n}=\left(\chi_{a n_{1}}+\chi_{a n_{2}}\right) / 2
$$

We note that the accuracy of the approximations (28) and (29) is dependent upon the specific material and operating conditions under consideration. To accommodate inaccuracies due to either these approximations or measurement errors, the following algorithms employ the remaining constraints to refine estimated parameter values. 


\subsubsection{Determination of Parameters}

The susceptibility and saturation criteria can be combined to provide algorithms for specifying the parameters $a, \alpha, k$ and $c$ and updating $P_{s}$. In certain cases, the relations provide implicit constraints on the variables which necessitates the use of root finding techniques (e.g., the Matlab routine fzero.m) for solution. Algorithms 1 and 2 differ in the manner through which parameter estimates are refined. In both cases, initial estimates are obtained directly from the constraints. In Algorithm 1, these estimates are refined by iterating through the constraints. While direct to implement, this technique does not enforce criteria which guarantee convergence. The second algorithm is more robust since refinement is accomplished through the minimization of a functional which simultaneously incorporates all constraints.

The accuracy of the parameters obtained using either method is dependent upon the degree to which the slope information at the initial, remanence, coercive and extreme points quantifies the overall behavior of the hysteresis curve. For cases in which this information is not sufficient, the parameter estimates can be employed as initial values in the least squares routine described in Section 3.2. This incorporates the full behavior of the hysteresis curve and produces model fits which are optimal in a least squares sense.

\section{Algorithm 1 (Iterative Refinement):}

\section{(A) Determine Initial Parameter Values:}

(1) Specify $c$ : From (22),

$$
c=\frac{\chi_{m}^{-}}{\chi_{m}^{+}} .
$$

This can be updated using $c=\chi_{i n} / \chi_{a n}$ from (18) using either measured values of $\chi_{i n}$ and $\chi_{a n}$ or approximates given by (28) and (29), respectively.

(2) Specify $a$ : From (20),

$$
a=\frac{P_{s}}{3 \chi_{a n}}
$$

where $\chi_{a n}$ is either measured or approximated using (29).

(3) Specify $\alpha$ : Solve (24)

$$
\chi_{r}=(1-c) \frac{P_{a n}\left(P_{r}\right)-P_{r}}{k \delta(1-c)-\alpha\left(P_{a n}\left(P_{r}\right)-P_{r}\right)}+c \frac{d P_{a n}\left(P_{r}\right)}{d E}
$$

using $k=E_{c}$ from (27).

(4) Specify $k$ : From (25)

$$
k=P_{a n}\left(E_{c}\right)\left[\frac{\alpha}{1-c}+\left(\frac{1}{\chi_{c}-c \frac{d P_{a n}\left(E_{c}\right)}{d E}}\right)\right] .
$$

(B) Iterative Refinement: Iterate until convergence is achieved.

(i) Solve (24) for $\alpha$

(ii) Solve (23) for $a$

(iii) Solve (25) for $k$ 


\section{Algorithm 2 (Simultaneous Refinement):}

(A) Determine Initial Parameter Values: Same as Algorithm 1.

(B) Simultaneous Refinement: Solve

$$
\min _{q}\|\mathcal{F}(q)\|
$$

where $q=[\alpha, a, c, k]$ and $\mathcal{F}(q)=\left[F_{1}(q), F_{2}(q), F_{3}(q)\right]^{T}$ with

$$
\begin{aligned}
& F_{1}(q)=\chi_{r}-(1-c) \frac{P_{a n}\left(P_{r}\right)-P_{r}}{k \delta(1-c)-\alpha\left(P_{a n}\left(P_{r}\right)-P_{r}\right)}-c \frac{d P_{a n}\left(P_{r}\right)}{d E} \\
& F_{2}(q)=k-P_{a n}\left(E_{c}\right)\left[\frac{\alpha}{1-c}+\left(\frac{1}{\chi_{c}-c \frac{d P_{a n}\left(E_{c}\right)}{d E}}\right)\right] \\
& F_{3}(q)=\chi_{m}^{+}-\frac{P_{a n}\left(E_{m}\right)-P_{m}}{k \delta(1-c)-\alpha\left(P_{a n}\left(E_{m}\right)-P_{m}\right)}
\end{aligned}
$$

Note: One can also consider $q=\left[\alpha, a, c, k, P_{s}\right]$ to additionally update $P_{s}$.

\subsection{Least Squares Determination of Parameters}

The algorithms developed in Section 3.1 highlight the physical nature of the parameters and are straightforward to implement. Because they incorporate a limited amount of information through the constraints, however, they may not provide sufficient accuracy in certain applications. A least squares method of the type described here incorporates the polarization values measured throughout the hysteresis cycle and can be used to obtain parameters that yield models which optimally fit the data in a least squares sense.

To formulate the least squares parameter estimation problem, let $\left(\widehat{E}_{i}, \widehat{P}_{i}\right), i=1, \cdots, \mathcal{N}$, denote the field and corresponding polarization values measured throughout the hysteresis cycle. Furthermore, let $P\left(\widehat{E}_{i} ; q\right)$ denote the parameter-dependent model solutions specified by (12). For admissible parameters $q \in Q$, we then solve the optimization problem

$$
\min _{q} \sum_{i=1}^{\mathcal{N}}\left|P\left(\widehat{E}_{i} ; q\right)-\widehat{P}_{i}\right|^{2} .
$$

An initial value $q_{0}$ can be specified either through a priori information or the parameter estimates obtained using the algorithm developed in the previous section. Model fits obtained using this procedure are provided in the next section. 


\section{Model Validation}

To illustrate the performance and prediction capabilities of the model and parameter estimation methods, we consider the characterization of hysteresis in a variety of PZT compounds. Specifically, we consider its performance for PZT5A, PZT5H, and PZT4 wafers as well as its flexibility for characterizing the hysteresis in patches having different geometries. The materials and geometric configurations which we consider are summarized in Table 1. All data was collected at $200 \mathrm{mHz}$ to maintain quasistatic operating conditions with the exception of a $1 \mathrm{~Hz}$ set for PZT5A which is included to illustrate that even at $1 \mathrm{~Hz}$, frequency-dependent effects are observed in the material.

The parameters predicted by Algorithm 2 in Section 3.1 and the least squares method from Section 3.2 are compiled in Table 2. The measured field, polarization and slope characteristics employed in the algorithms are summarized in Table 3. We note that measured values of the initial anhysteretic and normal polarization curves were not available so $\chi_{i n}$ and $\chi_{a n}$ were approximated using (28) and (29). A comparison of the parameters predicted by Algorithm 2 and the least squares method illustrates that while they are close, the least squares fit refines the values to provide better fits throughout the hysteresis cycle.

\section{Example 1 (PZT5A):}

We first consider the characterization of hysteresis exhibited by PZT5A actuators with three sets of data being considered. The first two illustrate that different actuators can exhibit slightly different hysteresis characteristics which necessitates the updating of parameters. The third illustrates that frequency-dependent effects can be present even at $1 \mathrm{~Hz}$ which motivates the use of $200 \mathrm{mHz}$ data for quasistatic material characterization.

\begin{tabular}{l|cccc} 
& Material & Frequency & Geometry & Dimensions \\
\hline \multirow{3}{*}{ Example 1 } & PZT5A & $200 \mathrm{mHz}$ & Circular & $2.54 \mathrm{~cm}$ Diameter, 0.0254 cm Thick \\
& PZT5A & $200 \mathrm{mHz}$ & Rectangular & $1.7 \mathrm{~cm} \times 0.635 \mathrm{~cm} \times 0.0381 \mathrm{~cm}$ \\
& PZT5A & $1 \mathrm{~Hz}$ & Rectangular & $1.7 \mathrm{~cm} \times 0.635 \mathrm{~cm} \times 0.0381 \mathrm{~cm}$ \\
\hline Example 2 & PZT5H & $200 \mathrm{mHz}$ & Rectangular & $3.81 \mathrm{~cm} \times 0.635 \mathrm{~cm} \times 0.0381 \mathrm{~cm}$ \\
\hline Example 3 & PZT4 & $200 \mathrm{mHz}$ & Rectangular & $3.81 \mathrm{~cm} \times 0.635 \mathrm{~cm} \times 0.0381 \mathrm{~cm}$ \\
\hline
\end{tabular}

Table 1. Materials, frequencies and geometrical configurations considered in the examples.

\begin{tabular}{|l|cc|cc|cc|cc|}
\hline & \multicolumn{2}{|c|}{ PZT5A $^{*}(1600 \mathrm{~V})$} & \multicolumn{2}{|c|}{ PZT5A $^{\dagger}(1600 \mathrm{~V})$} & \multicolumn{2}{c|}{ PZT5H (2200 V) } & \multicolumn{2}{c|}{ PZT4 (1800 V) } \\
& Alg. 2 & Least Sq. & Alg. 2 & Least Sq. & Alg. 2 & Least Sq. & Alg. 2 & Least Sq. \\
\hline$\alpha$ & $3.6 \times 10^{6}$ & $3.6 \times 10^{6}$ & $3.1 \times 10^{6}$ & $3.7 \times 10^{6}$ & $4.0 \times 10^{6}$ & $4.2 \times 10^{6}$ & $6.5 \times 10^{6}$ & $6.4 \times 10^{6}$ \\
$a$ & $4.4 \times 10^{5}$ & $4.2 \times 10^{5}$ & $4.2 \times 10^{5}$ & $4.1 \times 10^{5}$ & $5.8 \times 10^{5}$ & $6.4 \times 10^{5}$ & $8.3 \times 10^{5}$ & $8.0 \times 10^{5}$ \\
$k$ & $1.9 \times 10^{6}$ & $1.8 \times 10^{6}$ & $1.8 \times 10^{6}$ & $1.5 \times 10^{6}$ & $1.1 \times 10^{6}$ & $1.0 \times 10^{6}$ & $2.5 \times 10^{6}$ & $1.5 \times 10^{6}$ \\
$c$ & 0.18 & 0.30 & 0.22 & 0.15 & 0.14 & 0.20 & 0.37 & 0.40 \\
$P_{s}$ & 0.49 & 0.49 & 0.49 & 0.49 & 0.425 & 0.425 & 0.44 & 0.44 \\
\hline
\end{tabular}

Table 2. Parameters determined using Algorithm 2 and the least squares method of Section 3.2

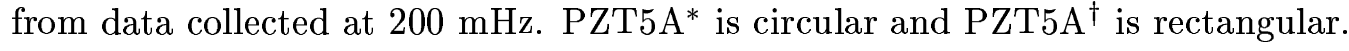




\begin{tabular}{|l|cccc|}
\hline & $\begin{array}{c}\text { PZT5A (Circular) } \\
(1600 \mathrm{~V})\end{array}$ & $\begin{array}{c}\text { PZT5A (Rectangular) } \\
(1600 \mathrm{~V})\end{array}$ & $\begin{array}{c}\text { PZT5H } \\
(2200 \mathrm{~V})\end{array}$ & $\begin{array}{c}\text { PZT4 } \\
(1800 \mathrm{~V})\end{array}$ \\
\hline$E_{c}(V / m)$ & $1.2 \times 10^{6}$ & $1.3 \times 10^{6}$ & $0.87 \times 10^{6}$ & $1.4 \times 10^{6}$ \\
$\chi_{c}(C /(m V))$ & $7.3 \times 10^{-7}$ & $7.2 \times 10^{-7}$ & $7.5 \times 10^{-7}$ & $1.2 \times 10^{-6}$ \\
$P_{r}\left(C / m^{2}\right)$ & 0.38 & 0.38 & 0.28 & 0.31 \\
$\chi_{r}(C /(m V))$ & $4.4 \times 10^{-8}$ & $5.7 \times 10^{-8}$ & $8.2 \times 10^{-8}$ & $4.2 \times 10^{-8}$ \\
$E_{m}(V / m)$ & $6.2 \times 10^{6}$ & $4.2 \times 10^{6}$ & $5.8 \times 10^{6}$ & $4.7 \times 10^{6}$ \\
$P_{m}\left(C / m^{2}\right)$ & 0.46 & 0.43 & 0.38 & 0.39 \\
$\chi_{m}^{+}(C /(m V))$ & $2.8 \times 10^{-8}$ & $3.1 \times 10^{-8}$ & $1.5 \times 10^{-8}$ & $2.5 \times 10^{-8}$ \\
$\chi_{m}^{-}(C /(m V))$ & $5.0 \times 10^{-9}$ & $6.7 \times 10^{-9}$ & $2.1 \times 10^{-9}$ & $9.5 \times 10^{-9}$ \\
\hline
\end{tabular}

Table 3. Coercive, remanence and tip characteristics measured from $200 \mathrm{mHz}$ data.

\section{Actuator 1 (Circular Patch):}

As summarized in Table 1, the circular wafer had a diameter of $2.54 \mathrm{~cm}$ and was $0.0254 \mathrm{~cm}$ (10 mils) thick. The wafer was depoled before use so the initial cycle contained transient behavior as the material was polarized. Three complete steady state cycles were then measured for input voltages ranging from $600 \mathrm{~V}$ to $1600 \mathrm{~V}$. The corresponding field inputs to the model were determined using the relation

$$
E=V / d
$$

where $d=0.0254 \mathrm{~cm}$ is the thickness of the wafer.

The parameters $\alpha, a, c, k$ and $P_{s}$ were estimated using both techniques discussed in Section 3. The employment of the data characteristics, summarized in Table 3, in Algorithm 2 provided the first set of parameter values listed in Table 2. We note that the asymptotic relations employed in this algorithm are more accurate near saturation which motivated the use of the $1600 \mathrm{~V}$ input data. The second set of values were obtained through a least squares fit to the $1600 \mathrm{~V}$ data. A comparison of parameter values obtained using the two techniques reveals a close match between the values for $\alpha, a$ and $k$ with some discrepancy in the values of $c$ due to limitations in determining $\chi_{m}^{+}$and $\chi_{m}^{-}$.

The modeled polarization was then computed using the Langevin anhysteretic expression and the parameter values determined through the least squares fit to $1600 \mathrm{~V}$ data for peak input voltages ranging from $600 \mathrm{~V}$ to $1600 \mathrm{~V}$. This model behavior is compared with the measured data in Figure 6 . We first note that the model fit for the $1600 \mathrm{~V}$ input is very accurate since this data was used to determine the parameters. Furthermore, a comparison between the model behavior at $600 \mathrm{~V}, 800 \mathrm{~V}$ and $1000 \mathrm{~V}$ indicates that throughout the range of drive levels, the model very accurately predicts the measured polarization. We reiterate that this capability of the model for predicting the polarization at various drive levels is due to the fact that it is based on energy principles.

We chose to employ the $1600 \mathrm{~V}$ data for the least squares algorithm solely to maintain consistency with the asymptotic Algorithm 2. A comparison with initial results presented in [20] illustrates that for this sample, equally accurate model fits and predictions at all drive levels can be obtained using the parameter values $\alpha=3.7 \times 10^{6} \mathrm{Vm} / \mathrm{C}, a=4.1 \times 10^{5} \mathrm{C} / \mathrm{m}^{2}, c=0.35, k=1.8 \times 10^{6} \mathrm{C} / \mathrm{m}^{2}$ and $P_{s}=0.49 \mathrm{C} / \mathrm{m}^{2}$ obtained through a least squares fit to the $600 \mathrm{~V}$ data. This again indicates the flexibility of the model due to its energy formulation. 

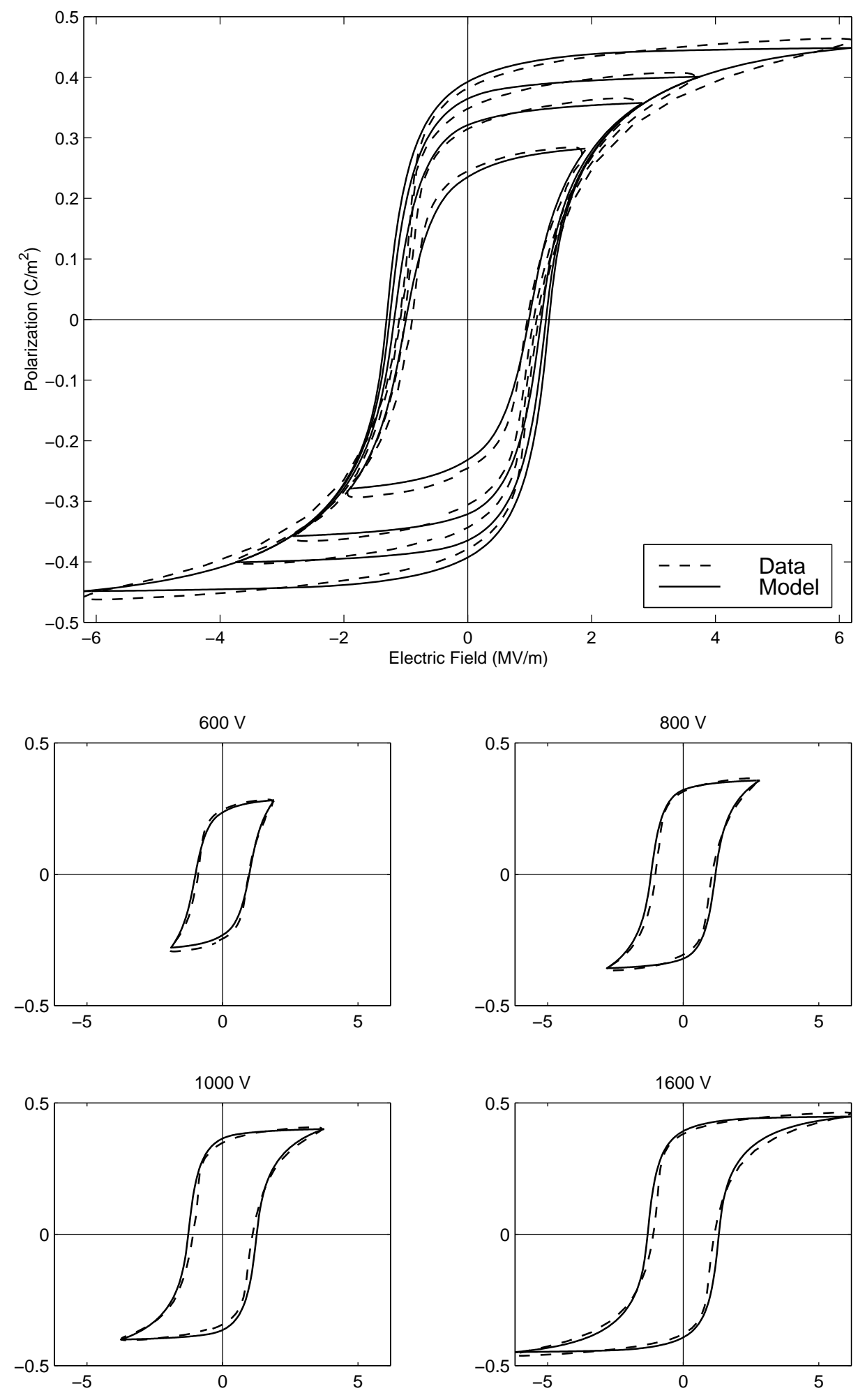

Figure 6. Model fit to $1600 \mathrm{~V}$ data for the circular PZT5A patch and model predictions at $600 \mathrm{~V}$, $800 \mathrm{~V}$ and $1000 \mathrm{~V}$ with the parameter choices $\alpha=3.6 \times 10^{6} \mathrm{Vm} / \mathrm{C}, a=4.2 \times 10^{5} \mathrm{C} / \mathrm{m}^{2}, c=0.30$, $k=1.8 \times 10^{6} \mathrm{C} / \mathrm{m}^{2}$ and $P_{s}=0.49 \mathrm{C} / \mathrm{m}^{2} ;$ Model $(-)$, Data $(---)$. 


\section{Actuator 2 (Rectangular Patch):}

The second PZT5A actuator which we considered was a rectangular patch having a thickness of $0.0381 \mathrm{~cm}$ ( $15 \mathrm{mils})$. We include this configuration to illustrate the variability which can occur between batches and the manner through which it affects the model. The data also illustrates certain frequency-dependencies which must be accommodated in broadband applications.

To illustrate the potential variability among actuators, the data collected from the rectangular patch with a peak input of $1800 \mathrm{~V}$ is compared in Figure $7 \mathrm{a}$ with $1200 \mathrm{~V}$ data from the 10 mil thick circular patch. While the field relation (31) indicates that the polarization should theoretically agree, the data illustrates a significant difference in the coercive field due to differences between the materials. This necessitates the refinement of parameters to attain accurate model fits throughout the range of operation.

The effect of even slight frequency shifts is illustrated in Figure $7 \mathrm{~b}$ where quasistatic data collected at $200 \mathrm{mHz}$ and a peak input voltage of $2200 \mathrm{~V}$ is compared with corresponding $1 \mathrm{~Hz}$ data. (Note that this is the only figure where $1 \mathrm{~Hz}$ data is included.) In its current state, the model parameters would require updating to accommodate the observed difference in saturation behavior. While this can be done if the frequency is fixed, updating in this manner relies on the mathematical rather than physical properties of the model. The extension of the model to physically incorporate frequency effects is under investigation.

The modeled polarization curves obtained with the updated parameters summarized in Table 2 are compared with the data in Figure 8. It is observed that as with the circular patch, the model accurately characterizes the hysteresis throughout the drive range of the material.

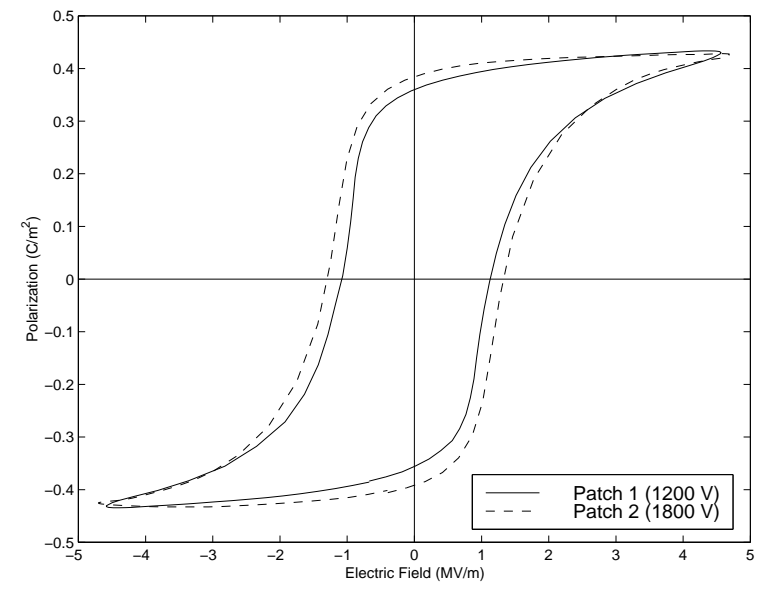

(a)

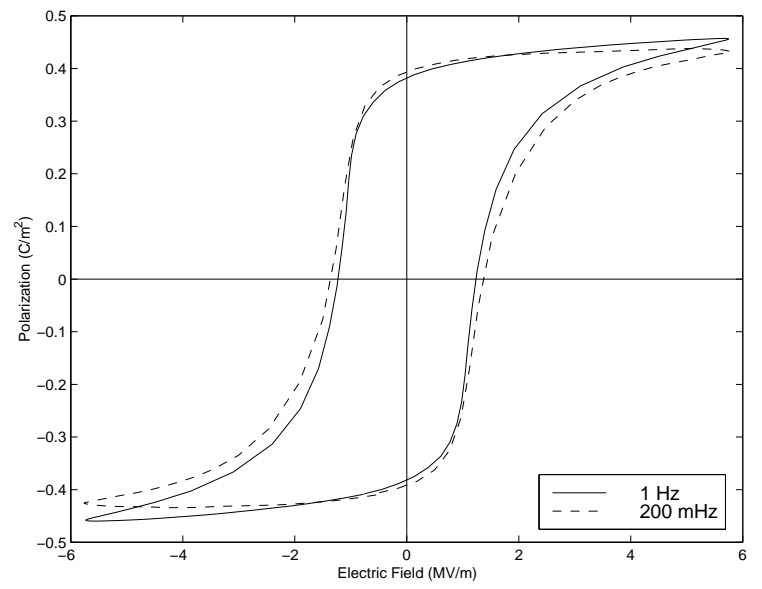

(b)

Figure 7. Comparison between $1200 \mathrm{~V}$ data from the $0.0254 \mathrm{~cm}(10 \mathrm{mil})$ circular patch and 1800 $\mathrm{V}$ data from the $0.0381 \mathrm{~cm}$ (15 mil) rectangular patch; (b) Comparison between $1 \mathrm{~Hz}$ and $200 \mathrm{mHz}$ data from the rectangular patch. 

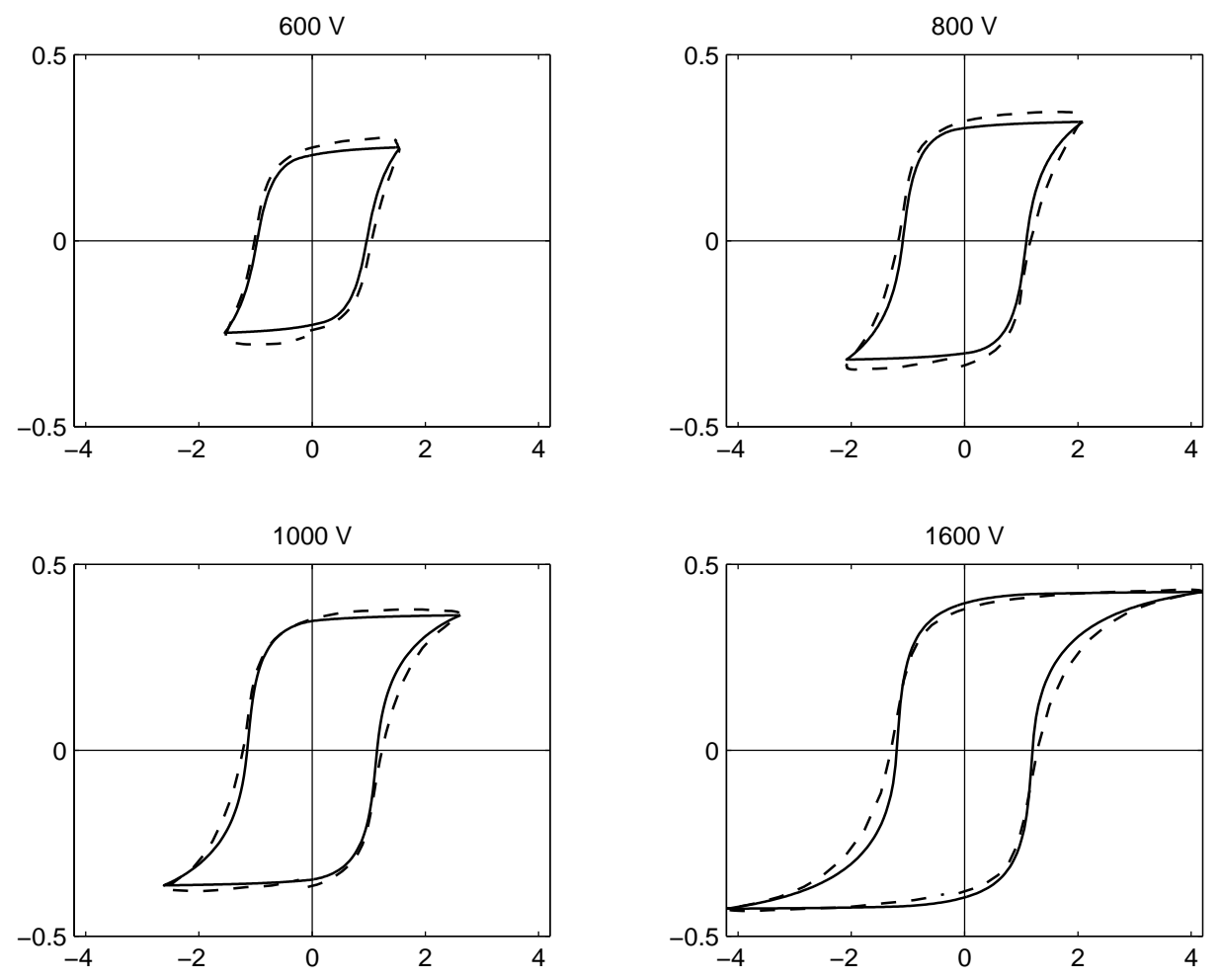

Figure 8. Model fit to $600,800,1000,1600 \mathrm{~V}$ data, collected at $200 \mathrm{mHz}$ from the rectangular PZT5A patch, with the parameter choices $c=0.15, k=1.5 \times 10^{6} \mathrm{C} / \mathrm{m}^{2}, \alpha=3.7 \times 10^{6} \mathrm{Vm} / \mathrm{C}$, $a=4.1 \times 10^{5} \mathrm{C} / \mathrm{m}^{2}$ and $P_{s}=.49 \mathrm{C} / \mathrm{m}^{2}$.

\section{Example 2 (PZT5H):}

A second commonly employed soft PZT material is PZT5H. In this example, we illustrate the performance of the model for a variety of parameter choices through a comparison with experimental data generated with input voltages of $600 \mathrm{~V}, 1000 \mathrm{~V}, 1600 \mathrm{~V}$ and $2200 \mathrm{~V}$.

The initial parameter values summarized in Table 2 were obtained using Algorithm 2 and the least squares algorithm with the $2200 \mathrm{~V}$ data. A comparison of the parameter values for $k$ and $c$ with those of PZT5A indicates smaller values for the PZT5H sample. The respective decreases in both the energy required to break pinning sites and the amount of reversible domain wall bending reflects the fact that PZT5H is softer than PZT5A as evidenced by the lower coercive field. We also note that for this soft material, the parameter $k$ is nearly equal to the coercivity $E_{c}$ as predicted by (27).

The model fits with the parameters obtained with the $2200 \mathrm{~V}$ data are compared with the data in Figure 9. It is observed that the model with parameters estimated using the least squares algorithm provides an excellent characterization throughout the hysteresis cycle whereas the model with parameters specified by Algorithm 2 accurately quantifies the slope at the remanence, coercive and saturation points but exhibits a slight discrepancy in polarization. This indicates the benefit of employing a least squares algorithm to obtain final parameter values.

The model with parameters obtained through a least squares fit to $2200 \mathrm{~V}$ data is then used to predict the polarization at lower drive levels with the resulting fits plotted in Figure 10. It is observed that with these parameters, the model provides accurate predictions down through $1000 \mathrm{~V}$ 
but degrades somewhat at $600 \mathrm{~V}$. For applications which require the full range of actuator operation, a second strategy is to consider the least squares fits to data from a variety of drive levels to provide parameters which optimize the model performance throughout the input range. This resulted in a modification of the value of $k$ from $k=1.0 \times 10^{6} \mathrm{C} / \mathrm{m}^{2}$ to $k=0.9 \times 10^{6} \mathrm{C} / \mathrm{m}^{2}$ and produced the model fits plotted in Figure 11. With this choice, the characterization at $2200 \mathrm{~V}$ is slightly less accurate but the model prediction at $600 \mathrm{~V}$ is improved. We reiterate that one set of parameters is still employed throughout the entire input range. In this case, however, the parameters have been optimized for the full operational range of the material.

Finally, we note that the parameter values predicted by Algorithm 2 using the $2200 \mathrm{~V}$ data are close to the values obtained through a least squares fit due to the accuracy of the asymptotic algorithm relations near saturation. To indicate the degradation in the accuracy of the relations which can occur at lower drive levels, we note using the $1000 \mathrm{~V}$ data, the algorithms produced the parameters $\alpha=3.6 \times 10^{6} \mathrm{Vm} / \mathrm{C}, a=4.3 \times 10^{5} \mathrm{C} / \mathrm{m}^{2}, k=1.2 \times 10^{6} \mathrm{C} / \mathrm{m}^{2}$ and $c=0.08$ which differ significantly from the values obtained near saturation or with the least squares fit. This indicates an advantage of the least squares method, which is independent of drive level, and the necessity of applying the asymptotic relations near saturation.

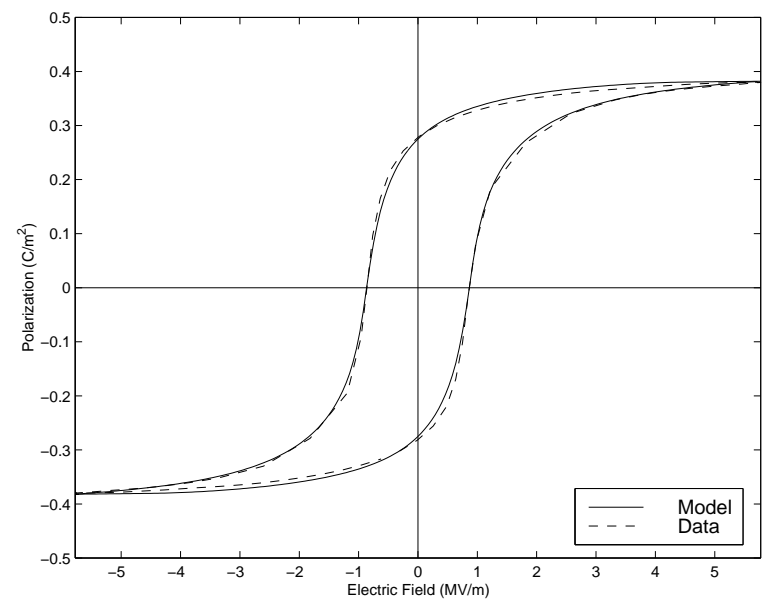

(a)

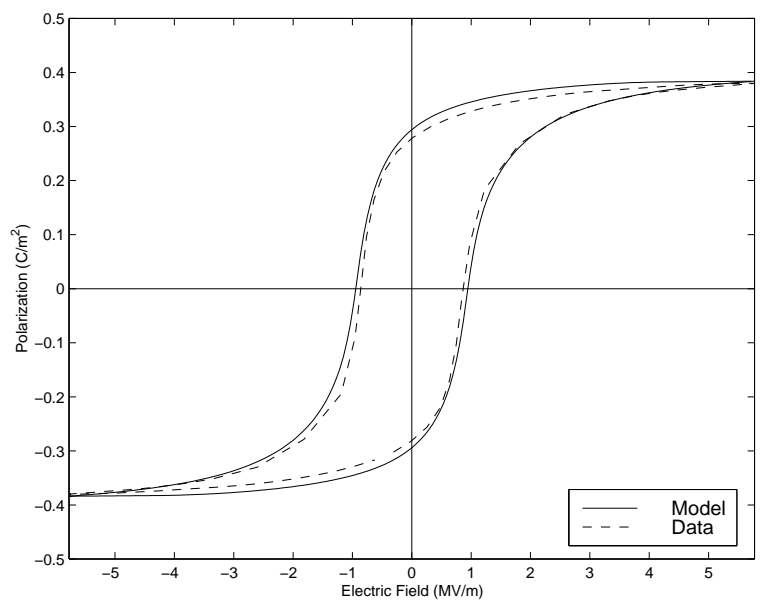

(b)

Figure 9. Model fits to the PZT5H data with parameters obtained through the least squares fit and Algorithm 2; (a) Least Squares: $\alpha=4.2 \times 10^{6} \mathrm{Vm} / \mathrm{C}, a=6.4 \times 10^{5} \mathrm{C} / \mathrm{m}^{2}, k=1.0 \times 10^{6} \mathrm{C} / \mathrm{m}^{2}, c=0.2$, $P_{s}=.425 \mathrm{C} / \mathrm{m}^{2}$, (b) Algorithm 2: $\alpha=4.0 \times 10^{6} \mathrm{Vm} / \mathrm{C}, a=5.8 \times 10^{5} \mathrm{C} / \mathrm{m}^{2}, k=1.1 \times 10^{6} \mathrm{C} / \mathrm{m}^{2}$, $c=0.14, P_{s}=.425 \mathrm{C} / \mathrm{m}^{2}$. 

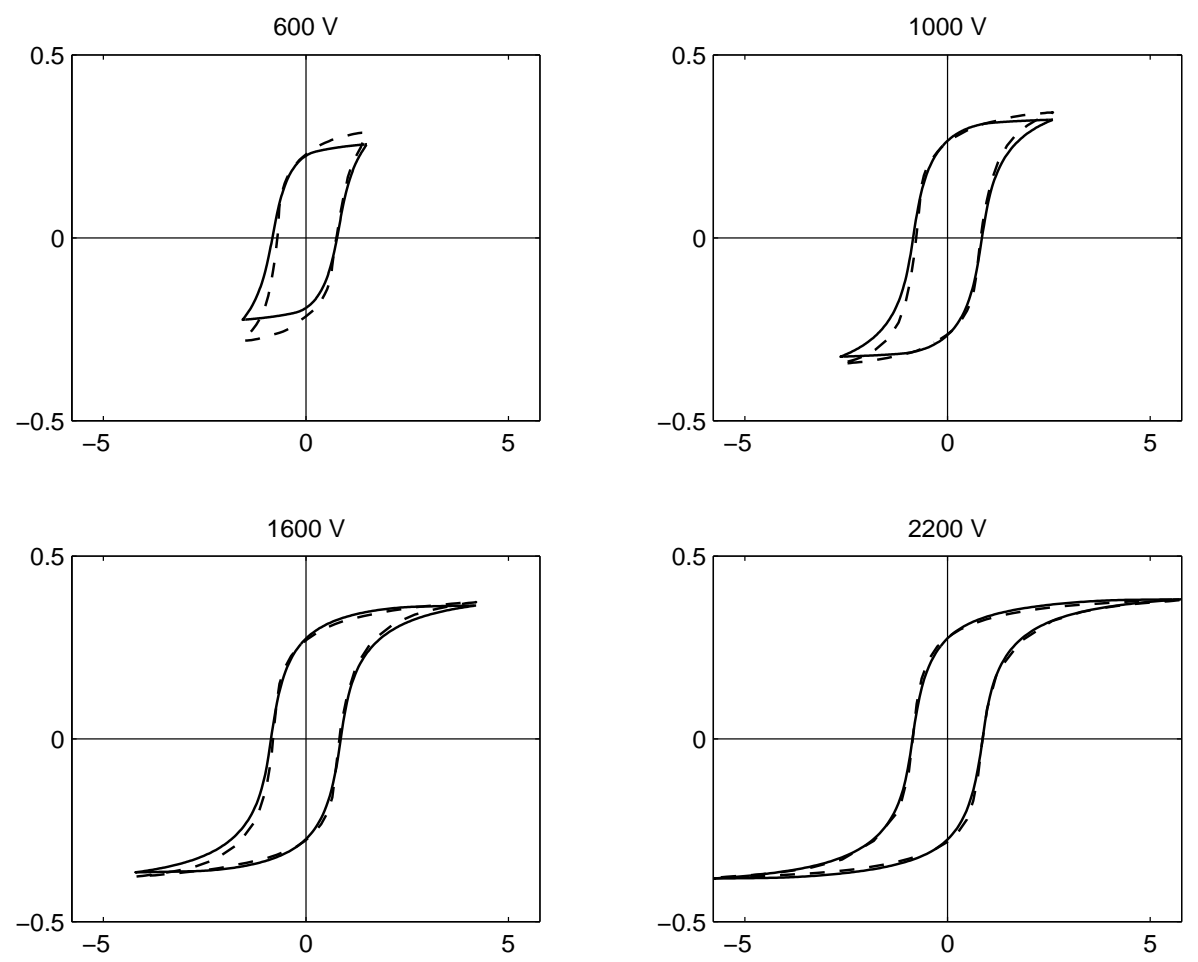

Figure 10. Model fit to the PZT5H data with the parameter choices $c=0.2, k=1.0 \times 10^{6} \mathrm{C} / \mathrm{m}^{2}$, $\alpha=4.2 \times 10^{6} \mathrm{Vm} / \mathrm{C}, a=6.4 \times 10^{5} \mathrm{C} / \mathrm{m}^{2}$ and $P_{s}=.425 \mathrm{C} / \mathrm{m}^{2}$.
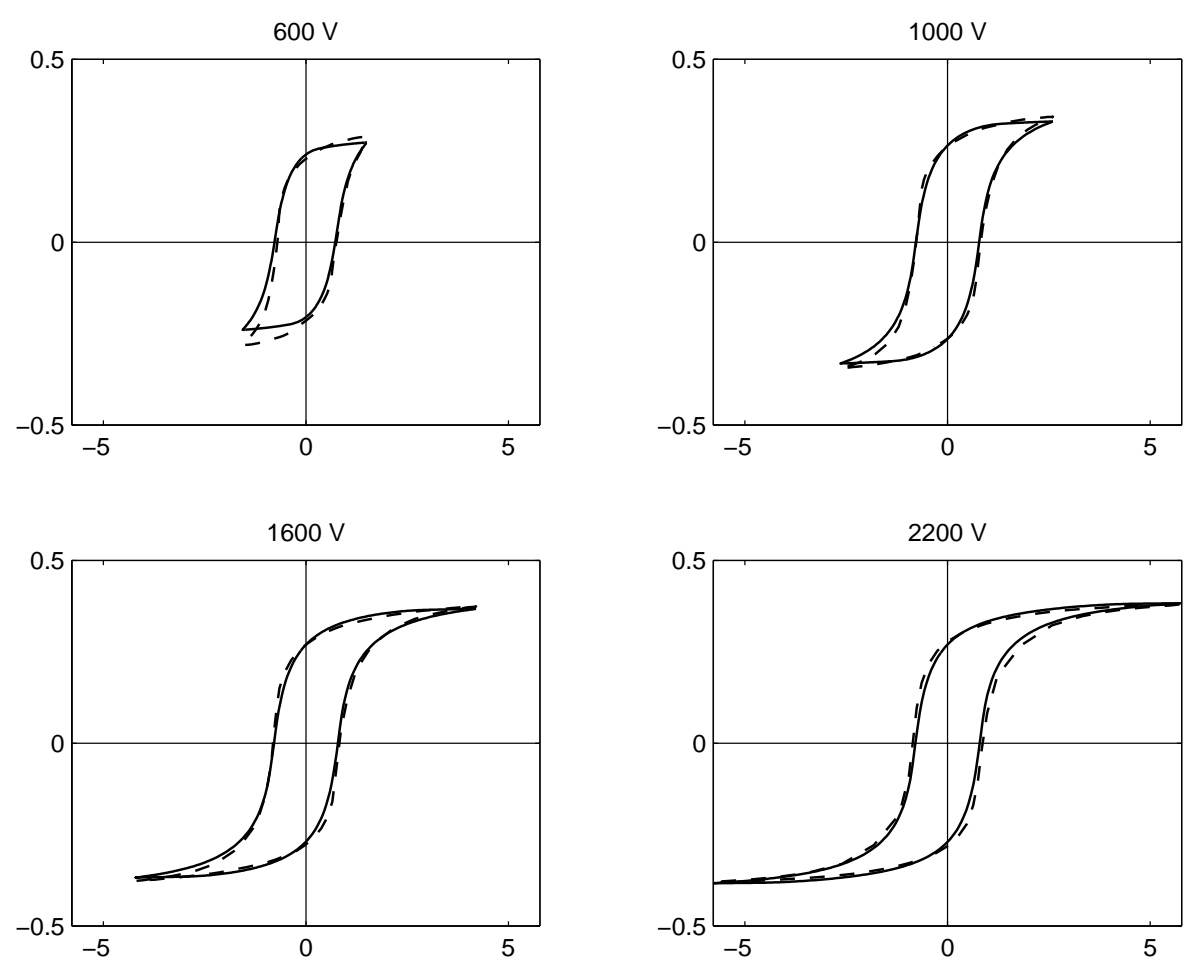

Figure 11. Model fit to the PZT5H data with the parameter choices $c=0.2, k=0.9 \times 10^{6} \mathrm{C} / \mathrm{m}^{2}$, $\alpha=4.2 \times 10^{6} \mathrm{Vm} / \mathrm{C}, a=6.4 \times 10^{5} \mathrm{C} / \mathrm{m}^{2}$ and $P_{s}=.425 \mathrm{C} / \mathrm{m}^{2}$. 


\section{Example 3 (PZT4):}

The final material that we consider is the harder compound PZT4 which is initially poled. Because more energy is required to turn dipoles, the material is not depoled when cycled, even at high input voltage levels. The remanent bias produces the asymmetry observed in Figure 3 and necessitates the inclusion of the bias field $E_{0}$ and polarization values $P_{0}$ and $P_{1}$ as indicated in (6) - (8). The bias can, however, be reduced if the material is maintained at a high voltage under thermally controlled conditions. The subsequent trajectories are nearly symmetric and exhibit minimal bias in field or polarization.

In this example, we illustrate the capability of the model to quantify both the biased (asymmetric) and unbiased (symmetric) polarization curves for PZT4. We consider first the characterization of biased data collected at a peak input voltage of $2200 \mathrm{~V}$. The data and model fit obtained with the parameter values $\alpha=6.4 \times 10^{6} \mathrm{Vm} / \mathrm{C}, a=8.0 \times 10^{5} \mathrm{C} / \mathrm{m}^{2}, k=1.5 \times 10^{6} \mathrm{C} / \mathrm{m}^{2}, c=0.5$, $P_{s}=.44 \mathrm{C} / \mathrm{m}^{2}$ and $E_{0}=-4.0 \times 10^{5} \mathrm{~V} / \mathrm{m}, P_{0}=.02 \mathrm{C} / \mathrm{m}^{2}, P_{1}=0$ are plotted in Figure 12 . It is observed that while the model is not able to completely quantify domain switching after positive remanence, it does characterize the primary behavior of the material throughout the cycle including the biased field and polarization and the associated asymmetry. The model with the same hysteresis parameters and $E_{0}=P_{0}=P_{1}$ is then compared to the nearly symmetric data, obtained after exposure to high input fields, in Figure 13. There it accurately predicts the polarization at $1000 \mathrm{~V}$, $1200 \mathrm{~V}$ and $1800 \mathrm{~V}$ input levels but under-predicts the hysteresis present at $600 \mathrm{~V}$. We note that these fits can be improved if the parameters are refined.

This example further illustrates the philosophy employed in this model. Through the incorporation of the associated physics, the model provides a characterization dependent only on inputs to the actuator and parameters quantifying the state of the material. Once the hysteresis parameters $\alpha, a, c, k$ and $P_{s}$ have been determined, the polarization obtained under quasistatic and isothermal operating conditions can be characterized using the measured input field and bias values $E_{0}$ and $P_{0}$. This provides the model with significant flexibility in a variety of applications.

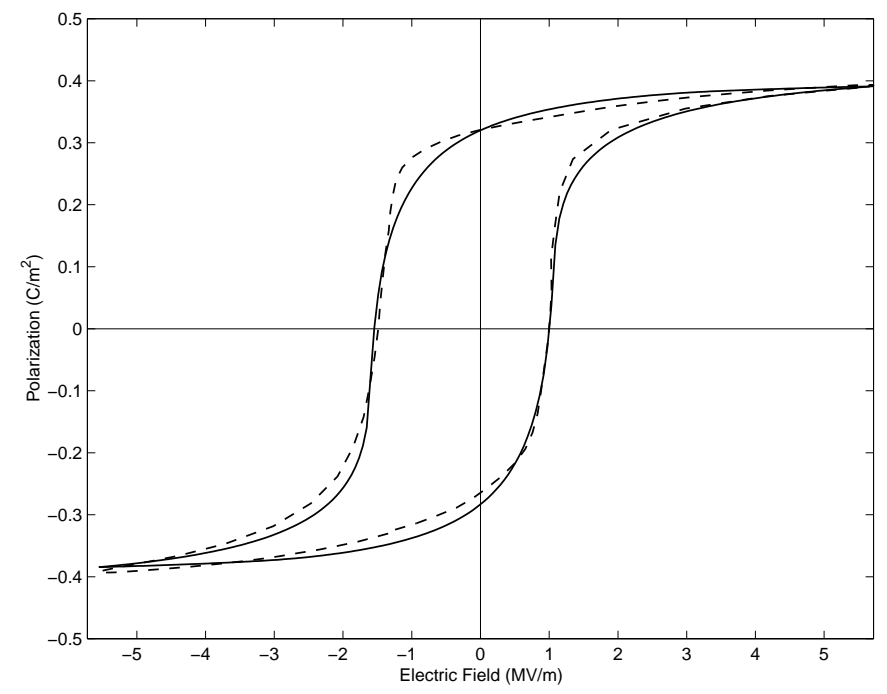

Figure 12. Model fit to the $2200 \mathrm{~V}$ PZT4 data with the parameters $\alpha=6.4 \times 10^{6} \mathrm{Vm} / \mathrm{C}$, $a=8.0 \times 10^{5} \mathrm{C} / \mathrm{m}^{2}, k=1.5 \times 10^{6} \mathrm{C} / \mathrm{m}^{2}, c=0.5, P_{s}=.44 \mathrm{C} / \mathrm{m}^{2}$ and $E_{0}=-4.0 \times 10^{5} \mathrm{~V} / \mathrm{m}$, $P_{0}=.02 \mathrm{C} / \mathrm{m}^{2}, P_{1}=0$. 

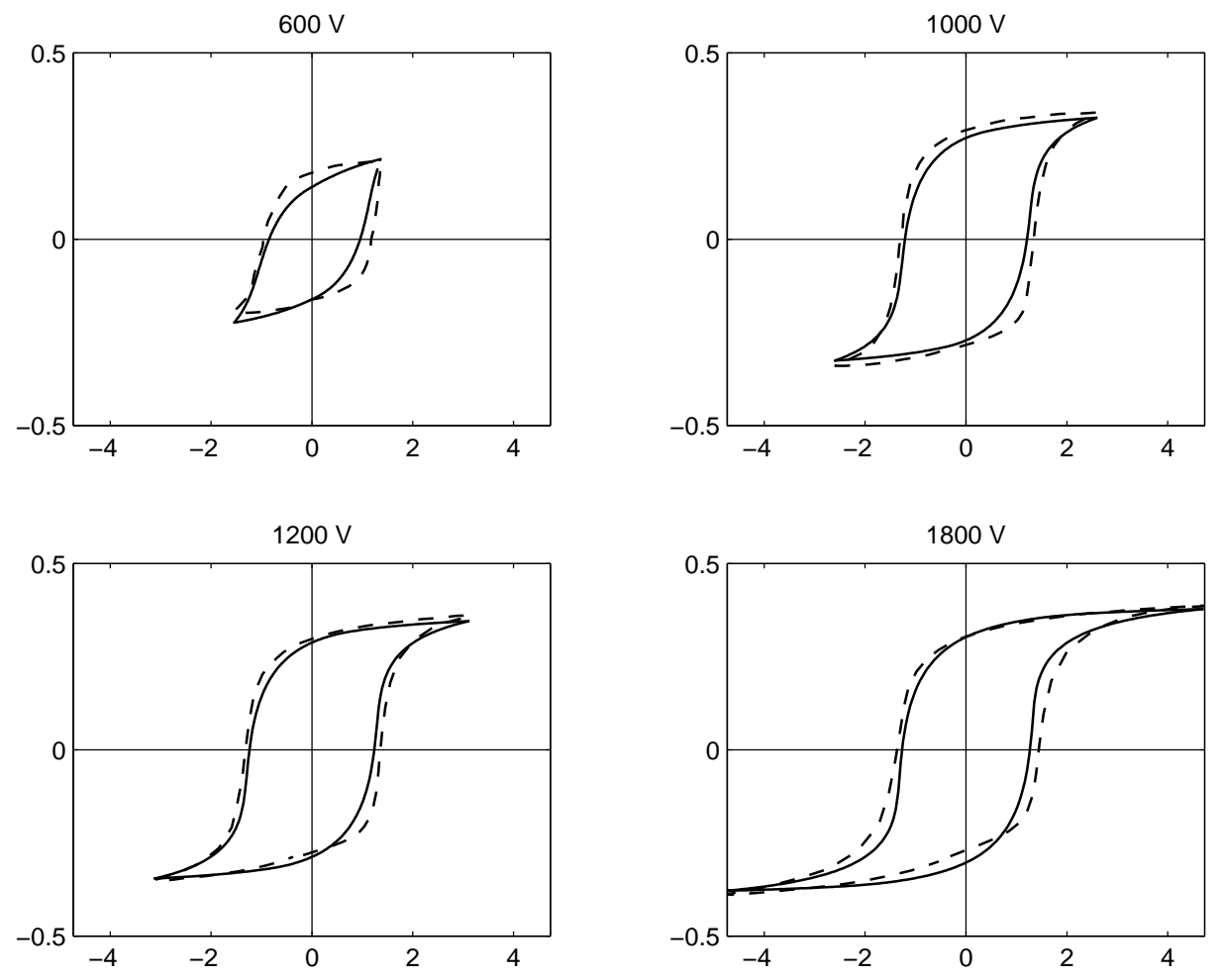

Figure 13. Model fit to PZT4 data with the parameters $\alpha=6.4 \times 10^{6} \mathrm{Vm} / \mathrm{C}, a=8.0 \times 10^{5} \mathrm{C} / \mathrm{m}^{2}$, $k=1.5 \times 10^{6} \mathrm{C} / \mathrm{m}^{2}, c=0.5, P_{s}=.44 \mathrm{C} / \mathrm{m}^{2}$ and $E_{0}=P_{0}=0$.

\section{Concluding Remarks}

This paper addresses the modeling of hysteresis in piezoelectric materials through the application and extension of a domain wall theory for ferroelectric materials [18, 19]. The theory characterizes the inherent hysteresis in the relation between the input field and output polarization through the quantification of energy required to bend and translate domain walls pinned at inclusions in the material. This provides reversible and irreversible polarization components whose sum represents the polarization due to an applied field. Characterization in this manner provides the model with the capability for specifying the polarization at a variety of input field levels with one set of model parameters. The flexibility of the model is further augmented by the small number (five) of required parameters and the physical nature of the parameters. For example, the saturation polarization $P_{s}$ is often known a priori or can be directly obtained from the data, the reversible coefficient $c$ can be estimated from the ratio of the slopes of the polarization curve at field reversal, and for soft materials, the pinning coefficient $k$ can be directly estimated from the coercive field $E_{c}$.

The contributions of this paper are threefold. The first focussed on the extension of the model to accommodate hysteresis loops which are not rotationally symmetric. This was motivated by the biasing effects observed in poled hard PZT actuators but the theory is sufficiently general to encompass a variety of applications. The second contribution was the validation of the theory and illustration of its predictive capabilities for three commonly employed PZT compounds. The theory in $[18,19]$ was illustrated only for PMN-PT-BT employed in the ferroelectric regime with no demonstration of the predictive capabilities, so this significantly extends the applicability of the theory. The third contributions of the paper was the development of asymptotic relations which 
highlight the physical nature of the five parameters and can be used to obtain initial parameter estimates. While the approach is analogous to that employed in [13] for ferromagnetic materials, the resulting relations and algorithms differ in certain respects.

The model, with parameters estimated using both the asymptotic relations and a least squares fit to the measured data, was used to characterize PZT5A, PZT5H and PZT4 actuators. In each case, the parameters were determined using data from one drive level. The model, with these parameters fixed, was then used to predict the polarization throughout the range of operation. As illustrated in the examples, the formulation of the model in terms of energy relations based on the input field provided it with the capability for prediction throughout the operational range. Furthermore, once the biasing field $E_{0}$ and polarization $P_{0}$ were determined, the model could accommodate the asymmetry exhibited by the poled PZT4. This provides the model with significant flexibility in a variety of applications.

In it current formulation, the theory is limited to quasistatic and thermally controlled operating regimes. Moreover, it was developed under the assumption that the effects of crystalline anisotropies are minimal. We note that in certain cases, parameters can be determined which provide accurate model fits at a variety of fixed frequencies. The use of the model in this manner relies on its mathematical rather than physical properties, however, which limits its flexibility and robustness with regard to changing dynamics. The extensions of the physical theory to accommodate the effects of frequency, transient temperatures and crystalline anisotropies are under current investigation.

Finally, the ODE nature of the model makes it amenable to inversion through the consideration of a complementary ODE in a manner analogous to that described in [17]. This facilitates the construction of an inverse compensator which can be used for linear control design [21, 22]. The application of these techniques for linear control implementation for piezoceramic actuators which exhibit hysteresis is under investigation.

\section{Acknowledgements}

The authors express sincere appreciation to Rory Schnell for her assistance in processing the data. The research of R.C.S. was supported in part by the Air Force Office of Scientific Research under the grant AFOSR-F49620-98-1-0180.

\section{References}

[1] R.M. Bozorth, Ferromagnetism, IEEE Press, Piscataway, NJ, 1978.

[2] W. Chen and C.S. Lynch, "A model for simulating polarization switching and AF-F phase changes in ferroelectric ceramics," Journal of Intelligent Material Systems and Structures, 9, pp. 427-431, 1998.

[3] I-W. Chen and Y. Wang, "A domain wall model for relaxor ferroelectrics," Ferroelectrics, 206, pp. 245-263, 1998.

[4] W. Elenbaas, "Relation between hysteresis curve and virgin curve of ferromagnetic substances," Physica, 12, pp. 125-132, 1932.

[5] V.N. Fedosov and A.S. Sidorkin, "Quasielastic displacements of domain boundaries in ferroelectrics," Soviet Physics Solid State, 18(6), pp. 964-968, 1976. 
[6] W.S. Galinaitis and R.C. Rogers, "Compensation for hysteresis using bivariate Preisach Models," SPIE Smart Structures and Materials, 1997, Mathematics and Control in Smart Structures, San Diego, CA, 1997.

[7] P. Ge and M. Jouaneh, "Modeling hysteresis in piezoceramic actuators," Precision Engineering, 17, pp. 211-221, 1995.

[8] P. Ge and M. Jouaneh, "Tracking control of a piezoceramic actuator," IEEE Transactions on Control Systems Technology, 4(3), pp. 209-216, 1996.

[9] L. Huang and H.F. Tiersten, "An analytic description of slow hysteresis in polarized ferroelectric ceramic actuators," Journal of Intelligent Material Systems and Structures, 9, pp. 417-426, 1998.

[10] B. Jaffe, W.R. Cook, Jr. and H. Jaffe, Piezoelectric Ceramics, Academic Press, New York, 1971.

[11] D.C. Jiles, Introduction to Magnetism and Magnetic Materials, Chapman and Hall, New York, 1991.

[12] D.C. Jiles and D.L. Atherton, "Theory of ferromagnetic hysteresis," Journal of Magnetism and Magnetic Materials, 61, pp. 48-60, 1986.

[13] D.C. Jiles, J.B. Thoelke and M.K. Devine, "Numerical determination of hysteresis parameters for the modeling of magnetic properties using the theory of ferromagnetic hysteresis," IEEE Trans. Magn., 28(1), pp. 27-35, 1992.

[14] B.D. Laikhtman, "Flexural vibrations of domain walls and dielectric dispersion of ferroelectrics," Soviet Physics Solid State, 15(1), pp. 62-68, 1973.

[15] M. Omura, H. Adachi and Y. Ishibashi, "Simulations of ferroelectric characteristics using a one-dimensional lattice model," Japanese Journal of Applied Physics, 30(9B), pp. 2384-2387, 1991.

[16] Piquette and S.E. Forsythe, "A nonlinear material model of lead magnesium niobate (PMN)," Journal of the Acoustical Society of America, 101(1), pp. 289-296, 1997.

[17] R.C. Smith, "Inverse compensation for hysteresis in magnetostrictive transducers," CRSC Technical Report CRSC-TR98-36; Mathematical and Computer Modeling, to appear.

[18] R.C. Smith and C.L. Hom, "A domain wall model for ferroelectric hysteresis," SPIE Conference on Mathematics and Control in Smart Structures, SPIE Volume 3667, Newport Beach, CA, March 1-4, 1999, pp. 150-161.

[19] R.C. Smith and C.L. Hom, "A domain wall theory for ferroelectric hysteresis," CRSC Technical Report CRSC-TR99-1; Journal of Intelligent Material Systems and Structures, to appear.

[20] R.C. Smith and Z. Ounaies, "A hysteresis model for piezoceramic materials," ICASE Report 99-29; Proceedings of the ASME International Mechanical Engineering Congress and Exposition, Nashville, TN, November, 1999, to appear.

[21] G. Tao and P.V. Kokotović, Adaptive Control of Systems with Actuator and Sensor Nonlinearities, John Wiley and Sons, New York, 1996.

[22] B. Widrow and E. Walach, Adaptive Inverse Control, Prentice Hall, NJ, 1996. 
[23] X.D. Zhang and C.A. Rogers, "A macroscopic phenomenological formulation for coupled electromechanical effects in piezoelectricity," Journal of Intelligent Material Systems and Structures, 4, pp. 307-316, 1993. 